\title{
From Principal Series to Finite-Dimensional Solutions of the Yang-Baxter Equation
}

\author{
Dmitry CHICHERIN ${ }^{\dagger}$, Sergey E. DERKACHOV ${ }^{\ddagger}$ and Vyacheslav P. SPIRIDONOV ${ }^{\S}$
}

† LAPTH, UMR 5108 du CNRS, associée à l'Université de Savoie, Université de Savoie, CNRS, B.P. 110, F-74941 Annecy-le-Vieux, France

E-mail: chicherin@lapth.cnrs.fr

$\ddagger$ St. Petersburg Department of Steklov Mathematical Institute of Russian Academy of Sciences, Fontanka 27, 191023 St. Petersburg, Russia

E-mail: derkach@pdmi.ras.ru

$\S$ Laboratory of Theoretical Physics, JINR, Dubna, Moscow region, 141980, Russia

E-mail: spiridon@theor.jinr.ru

Received November 17, 2015, in final form March 04, 2016; Published online March 11, 2016

http://dx.doi.org/10.3842/SIGMA.2016.028

\begin{abstract}
We start from known solutions of the Yang-Baxter equation with a spectral parameter defined on the tensor product of two infinite-dimensional principal series representations of the group $\mathrm{SL}(2, \mathbb{C})$ or Faddeev's modular double. Then we describe its restriction to an irreducible finite-dimensional representation in one or both spaces. In this way we obtain very simple explicit formulas embracing rational and trigonometric finite-dimensional solutions of the Yang-Baxter equation. Finally, we construct these finite-dimensional solutions by means of the fusion procedure and find a nice agreement between two approaches.
\end{abstract}

Key words: Yang-Baxter equation; principal series; modular double; fusion

2010 Mathematics Subject Classification: 81R50; 82B23; 33D05

\section{Contents}

1 Introduction $\quad 2$

$2 \mathrm{SL}(2, \mathbb{C})$ group $\quad 4$

2.1 Representations of the group and the intertwining operator $\ldots \ldots \ldots \ldots$

2.2 The general $\mathrm{SL}(2, \mathbb{C})$-invariant R-operator $\ldots \ldots \ldots \ldots \ldots \ldots$

2.3 Finite-dimensional reductions of the general R-operator $\ldots \ldots \ldots \ldots$

2.4 Verma module reduction . . . . . . . . . . . . . . . . . . . . . 11

2.5 Fusion, symbols and the Jordan-Schwinger representation . . . . . . . . . . . . . 13

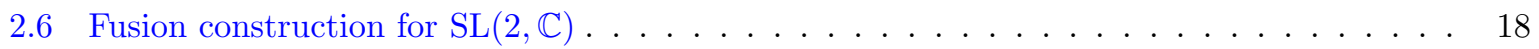

3 The Faddeev modular double $\quad 19$

3.1 Representations of the quantum algebra . . . . . . . . . . . . . . . . . . . . 19

3.2 An infinite-dimensional R-operator for the modular double . . . . . . . . . . . . . 23

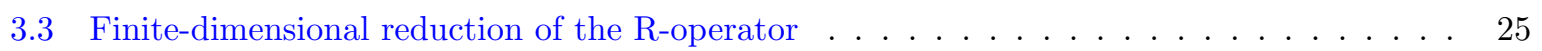

3.4 The fusion and symbols for $\mathcal{U}_{q}\left(\mathfrak{s l}_{2}\right)$ algebra $\ldots \ldots \ldots \ldots \ldots \ldots \ldots$

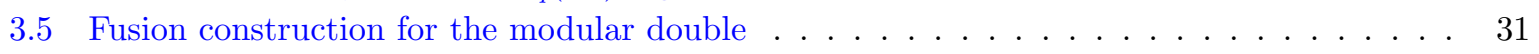

$\begin{array}{ll}\text { References } & 33\end{array}$ 
D. Chicherin, S.E. Derkachov and V.P. Spiridonov

\section{Introduction}

The Yang-Baxter equation (YBE)

$$
\mathbb{R}_{12}(u-v) \mathbb{R}_{13}(u) \mathbb{R}_{23}(v)=\mathbb{R}_{23}(v) \mathbb{R}_{13}(u) \mathbb{R}_{12}(u-v)
$$

is a major tool in building the quantum integrable systems [1, 26, 28, 29, 39]. It has found numerous applications in mathematical physics and purely mathematical questions. At the dawn of quantum inverse scattering method the finite-dimensional solutions of the YBE (when the operators $\mathbb{R}_{i j}(u)$ are given by ordinary matrices with numerical entries depending on the spectral parameter $u$ ) attracted much attention in view of their relevance for physical spin systems on lattices admitting a successful treatment of their thermodynamical behavior $[1,26]$.

Solutions of the YBE for infinite-dimensional representations revealed their importance in the integrability phenomena emerging in quantum field theories. An integrable spin chain with underlying $\mathrm{SL}(2, \mathbb{C})$ symmetry group and its noncompact representations naturally arises in the high-energy behavior of quantum chromodynamics. Corresponding model was discovered in [30] together with an additional integral of motion. Later, in [31] and [21] it was identified with the noncompact XXX spin chain which revealed its complete integrability (for further investigations of this model, see $[12,14])$.

There are three increasing levels of complexity of finite-dimensional solutions of YBE described by matrices with the coefficients expressed in terms of the rational, trigonometric, and elliptic functions. In the infinite-dimensional setting the latter hierarchy is replaced by solutions of YBE defined as integral operators with the integrands described by plain hypergeometric, $q$-hypergeometric and elliptic hypergeometric functions [36].

The notion of the modular double was introduced by Faddeev in [18] and noncompact representations of this algebra arise naturally in the Liouville model studies [20, 35]. The quantum dilogarithm function [19] plays an important role in the description of these representations as well as in the Faddeev-Volkov model [2,43] and its generalization found in [38]. The elliptic modular double extending Faddeev's double was introduced in [37].

The general solution of YBE at the elliptic level with the rank 1 symmetry algebra was found in [16]. It is based on the properties of an integral operator with an elliptic hypergeometric kernel, the key identity for which (given by the Bailey lemma, see, e.g., [36]) coincides with the star-triangle relation. In $[16,17]$ a particular finite-dimensional invariant space for the representations of the elliptic modular double has been described.

The general R-operator is interesting on its own. In the case of group $\mathrm{SL}(2, \mathbb{C})$ and the Faddeev and elliptic modular doubles it is represented by an explicit integral operator acting on the tensor product of two functional spaces $[6,14,15,16]$. It can be thought of as a universal object since it is expected that in some sense it conceals all solutions of YBE, particularly, the finite-dimensional solutions. In this paper we show explicitly that, indeed, the latter solutions can be derived as reductions of the infinite-dimensional R-operators in three particular cases: the $\mathrm{SL}(2, \mathbb{C})$ group R-operator [15], its real form analogue associated with the $\mathfrak{s l}_{2}$-algebra and the R-operator for the Faddeev modular double, which was considered first in [5] as a formal function with an operator argument.

Reductions to finite-dimensional invariant subspaces constitute a nontrivial problem. Indeed, general infinite-dimensional R-matrices are given by integral operators, but their reduction to a finite-dimensional invariant subspace in one of the tensor product spaces should be a matrix with the entries described by differential or finite-difference operators.

Our key results are given by the remarkably compact formulas for reduced R-operators (2.32), (2.38), and (3.36). The former and the latter cases are determined by a pair of integer parameters. In the $\mathrm{SL}(2, \mathbb{C})$-case $(2.32)$ two integers emerge from the discretization of two spin variables, $s$ and $\bar{s}$. In the modular double case (3.36) the situation is qualitatively different, two integers 
emerge from the intrinsically two-dimensional nature of the discrete lattice for one spin variable. In the context of univariate spectral problems such a quantization leads to the two-index orthogonality relations which were found for the first time in the theory of elliptic hypergeometric functions, see [36] and references therein. In our problem, two integers appearing in the reduction of R-matrix associated with the modular double are descendants from the similar integers existing at the elliptic level $[16,17]$.

It is well known that quantum integrable systems are related to $6 j$-symbols of different algebras. In the context of $2 d$ conformal field theory these symbols are associated with the fusion matrices and, in this setting, the finite-dimensional $6 j$-symbols of the modular double with $q$ a root of unity have been constructed in [22]. Their continuous spin generalizations have been built in [35]. The most general discrete $q-6 j$-symbols of such type (with the doubling of indices) are composed out of the product of two particular terminating ${ }_{10} \varphi_{9}$ basic hypergeometric series related by a modular transformation [36]. Their noncompact analogues associated with the lattice model of [38] and generalizing $6 j$-symbols of [35] are easily derived as a limiting case of the elliptic analogue of the Euler-Gauss hypergeometric function [36]. A similar set of questions was discussed recently for the quantum algebra $U_{q}(\mathfrak{o s p}(1 \mid 2))$ [34].

A conventional method of constructing higher spin L-operators or the higher spin R-matrices which are finite-dimensional in both spaces is the fusion procedure [28, 29]. It is based on the fact that arbitrary finite-dimensional representation of a rank 1 algebra is contained in the decomposition of a tensor power of the fundamental representation. Similarly, by means of the fusion procedure one constructs higher quantized spin solutions of YBE out of the fundamental one. In particular, a higher spin R-operator, which is finite-dimensional in one of the spaces, is given by a symmetrized tensor product of several Lax operators, and higher spin ordinary matrix solutions of YBE are given by symmetrized tensor products of several fundamental R-matrices.

There is another method of building such R-operators based on the observation that for special values of the spins (representation parameters) the principal series representation becomes reducible and a finite-dimensional irreducible representation decouples. The general R-operator does not map out of this invariant finite-dimensional subspace, so it can be restricted to this subspace and get a reduced form. In this approach the intertwining operators of equivalent representations of the symmetry algebras play a crucial role. They explicitly indicate specific values of the spin when such a decoupling takes place.

In this work we elaborate both methods for the $\mathrm{SL}(2, \mathbb{C})$ group and the modular double (the corresponding intertwining operators were constructed in [23] and [35]). We show explicitly that both methods yield identical formulae embracing required finite-dimensional (in one or both spaces) solutions of YBE. Additionally, we consider a finite-dimensional reduction of the R-operators for a tensor product of two Verma modules. This is the first paper in the series dedicated to finite-dimensional reductions of known integral R-operators. In the next work of this series [10] such a problem was solved for the elliptic modular double. In [7] new compact factorization formulae were derived for finite-dimensional R-matrices in several cases (for different forms of factorizations, see [27] and references therein). Reduction of the integral R-operator for the generalized Faddeev-Volkov model of [38] is considered in [11].

The paper consists of two parts. In the first part we consider $\mathrm{SL}(2, \mathbb{C})$-invariant solutions of YBE. We begin in Section 2.1 with a concise review of the infinite-dimensional principal series representation of the $\mathrm{SL}(2, \mathbb{C})$ group. In Section 2.2 we indicate the relevant Lax operators and the general R-operator emphasizing the role of the star-triangle relation. In Section 2.3 we reduce the general $\mathrm{SL}(2, \mathbb{C})$-symmetric R-operator to a finite-dimensional representation in one of the spaces. In Section 2.4 we derive an analogous reduction for the general $\mathfrak{s l}_{2}$-algebra R-operator to the space of polynomials or the Verma module.

Then we proceed to the fusion. In Section 2.5 we formulate the fusion for the $\mathfrak{s l}_{2}$ algebra case in a rather nonstandard fashion. We construct projectors to the highest spin representation by 
means of some auxiliary spinor variables that results in the Jordan-Schwinger realization of the "fused" representation. We describe also how the fusion procedure reproduces the L-operator as well. After that, in Section 2.6 we get back to the $\mathrm{SL}(2, \mathbb{C})$ group and carry out the fusion in this case.

In the second part of the paper we consider similar questions for the modular double. There the presentation closely follows the rational case in order to emphasize the striking similarity between these two cases. In Sections 3.1 and 3.2 we outline general structure of the modular double and present the general R-operator for it. The corresponding reduced R-matrix, which is finite-dimensional in one of the quantum spaces (or both), is derived in Section 3.3. Finally, in Sections 3.4 and 3.5 we derive finite-dimensional R-matrices in the $q$-deformed cases using the fusion procedure.

\section{$2 \mathrm{SL}(2, \mathbb{C})$ group}

\subsection{Representations of the group and the intertwining operator}

We start with a short review of some basic well-known facts about representations of the group $\mathrm{SL}(2, \mathbb{C})$. They are formulated in a form that will be natural for dealing with R-operators. We outline how finite-dimensional representations decouple from infinite-dimensional ones emphasizing the role of the intertwining operator.

The method of induced representations is a robust tool that enables one to construct a number of interesting representations of a group (see for example [24]). Consider representations of the group $\operatorname{SL}(2, \mathbb{C})$ realized on the space of single-valued functions $\Phi(z, \bar{z})$ on the complex plane. The principal series representation [23] is parametrized by a pair of generic complex numbers $(s, \bar{s})$ subject to the constraint $2(s-\bar{s}) \in \mathbb{Z}$. We refer to them as spins in what follows. In order to avoid misunderstanding we emphasize that $s$ and $\bar{s}$ are not complex conjugates in general. So, this representation $\mathrm{T}^{(s, \bar{s})}$ is given explicitly as [23]

$$
\begin{aligned}
& {\left[\mathrm{T}^{(s, \bar{s})}(g) \Phi\right](z, \bar{z})=(d-b z)^{2 s}(\bar{d}-\bar{b} \bar{z})^{2 \bar{s}} \Phi\left(\frac{-c+a z}{d-b z}, \frac{-\bar{c}+\bar{a} \bar{z}}{\bar{d}-\bar{b} \bar{z}}\right),} \\
& g=\left(\begin{array}{ll}
a & b \\
c & d
\end{array}\right) \in \operatorname{SL}(2, \mathbb{C}) .
\end{aligned}
$$

Representations of the group $\mathrm{SL}(2, \mathbb{C})$ yield representations of the Lie algebra $\mathfrak{s l}(2, \mathbb{C})$ in a standard way. Assuming that $g$ lies in a vicinity of the identity $g=1+\epsilon \cdot \mathcal{E}_{i k}$, where $\mathcal{E}_{i k}$ are traceless $2 \times 2$ matrices: $\left(\mathcal{E}_{i k}\right)_{j l}=\delta_{i j} \delta_{k l}-\frac{1}{2} \delta_{i k} \delta_{j l}$, one extracts generators $\mathrm{E}_{i k}$ and $\overline{\mathrm{E}}_{i k}$ of the Lie algebra,

$$
\mathrm{T}^{(s, \bar{s})}\left(1+\epsilon \cdot \mathcal{E}_{i k}\right) \Phi(z, \bar{z})=\Phi(z, \bar{z})+\left(\epsilon \cdot \mathrm{E}_{i k}+\bar{\epsilon} \cdot \overline{\mathrm{E}}_{i k}\right) \Phi(z, \bar{z})+O\left(\epsilon^{2}\right) .
$$

The generators $\mathrm{E}_{i k}, \overline{\mathrm{E}}_{i k}$ are the first-order differential operators. We arrange them in $2 \times 2$ matrices $\mathrm{E}^{(s)}$ and $\overline{\mathrm{E}}^{(\bar{s})}$, which will be useful for the following considerations,

$$
\mathrm{E}^{(s)}=\left(\begin{array}{ll}
\mathrm{E}_{11} & \mathrm{E}_{21} \\
\mathrm{E}_{12} & \mathrm{E}_{22}
\end{array}\right)=\left(\begin{array}{cc}
z \partial-s & -\partial \\
z^{2} \partial-2 s z & -z \partial+s
\end{array}\right)=\left(\begin{array}{cc}
1 & 0 \\
z & 1
\end{array}\right)\left(\begin{array}{cc}
-s-1 & -\partial \\
0 & s
\end{array}\right)\left(\begin{array}{cc}
1 & 0 \\
-z & 1
\end{array}\right) .
$$

The substitution $z \rightarrow \bar{z}, \partial \rightarrow \bar{\partial}$ and $s \rightarrow \bar{s}$ in this formula results in the matrix $\overline{\mathrm{E}}^{(\bar{s})}$ for the generators $\overline{\mathrm{E}}_{i k}$.

There exists an integral operator $\mathrm{W}$ which intertwines a pair of principal series representations $\mathrm{T}^{(s, \bar{s})}$ and $\mathrm{T}^{(-1-s,-1-\bar{s})}$ for generic complex $s$ and $\bar{s}$,

$$
\mathrm{W}(s, \bar{s}) \mathrm{T}^{(s, \bar{s})}(g)=\mathrm{T}^{(-1-s,-1-\bar{s})}(g) \mathrm{W}(s, \bar{s}) .
$$


We will refer to this pair as the equivalent representations. The described intertwining relation can be equally reformulated as a set of intertwining relations for the Lie algebra generators

$$
\mathrm{W}(s, \bar{s}) \mathrm{E}^{(s)}=\mathrm{E}^{(-1-s)} \mathrm{W}(s, \bar{s}), \quad \mathrm{W}(s, \bar{s}) \overline{\mathrm{E}}^{(\bar{s})}=\overline{\mathrm{E}}^{(-1-\bar{s})} \mathrm{W}(s, \bar{s}) .
$$

The operator $\mathrm{W}$ is defined up to an overall normalization and has the following explicit form [23]

$$
[\mathrm{W}(s, \bar{s}) \Phi](z, \bar{z})=\mathrm{const} \int_{\mathbb{C}} d^{2} x \frac{\Phi(x, \bar{x})}{(z-x)^{2 s+2}(\bar{z}-\bar{x})^{2 \bar{s}+2}} .
$$

Obviously this integral operator is well-defined for generic values of $s$ and $\bar{s}$ and the problems emerge for the discrete set of points $2 s=n, 2 \bar{s}=\bar{n}$ with $n, \bar{n} \in \mathbb{Z}_{\geq 0}$. These special values of the spins correspond to finite-dimensional representations which we are aiming at. That is why we would like to have a meaningful intertwining operator for this discrete set. In order to obtain it we note that the expression (2.5), considered as an analytical function of $s, \bar{s}$, has simple poles exactly on this discrete set of (half)-integer points. Consequently, we need to choose properly the normalization constant in (2.5) to suppress the poles at $2 s=n, 2 \bar{s}=\bar{n}$. Further, pursuing this strategy we find the normalization constant as an appropriate combination of the Euler gamma functions such that the intertwining operator (2.5) becomes well-defined in the case of finite-dimensional representations as well. In order to implement the outlined program we resort to the text-book formula for the following complex Fourier transformation [23]

$$
A(\alpha, \bar{\alpha}) \int_{\mathbb{C}} d^{2} z \frac{\mathrm{e}^{i p z+i \bar{p} \bar{z}}}{z^{1+\alpha} \bar{z}^{1+\bar{\alpha}}}=p^{\alpha} \bar{p}^{\bar{\alpha}}, \quad A(\alpha, \bar{\alpha})=\frac{i^{-|\alpha-\bar{\alpha}|}}{\pi} \frac{\Gamma\left(\frac{\alpha+\bar{\alpha}+|\alpha-\bar{\alpha}|+2}{2}\right)}{\Gamma\left(\frac{-\alpha-\bar{\alpha}+|\alpha-\bar{\alpha}|}{2}\right)},
$$

where $\Gamma(x)$ is the Euler gamma function. One can substitute here $z=x+i y, \bar{z}=x-i y$ and pass to the integrations over $x, y \in \mathbb{R}$. We replace $p$ and $\bar{p}$ by the differential operators, $p \rightarrow i \partial_{x}$ and $\bar{p} \rightarrow i \partial_{\bar{x}}$, use the shift operator $\mathrm{e}^{a \partial_{x}} f(x)=f(x+a)$, and come to the definition

$$
\left(i \partial_{z}\right)^{\alpha}\left(i \partial_{\bar{z}}\right)^{\bar{\alpha}} \Phi(z, \bar{z}):=A(\alpha, \bar{\alpha}) \int_{\mathbb{C}} d^{2} x(z-x)^{-1-\alpha}(\bar{z}-\bar{x})^{-1-\bar{\alpha}} \Phi(x, \bar{x}) .
$$

In order to avoid cumbersome expressions we prefer to recast this formula to a concise form

$$
\left[i \partial_{z}\right]^{\alpha} \Phi(z, \bar{z})=A(\alpha) \int_{\mathbb{C}} d^{2} x[z-x]^{-1-\alpha} \Phi(x, \bar{x}) .
$$

Here and in the following we profit from the shorthand notation

$$
[z]^{\alpha}=z^{\alpha} \bar{z}^{\bar{\alpha}}, \quad \alpha-\bar{\alpha} \in \mathbb{Z}
$$

which unifies the holomorphic and antiholomorphic sectors. Let us remind once more that $\alpha$ and $\bar{\alpha}$ are not assumed to be complex conjugates. The constraint on the exponents $\alpha, \bar{\alpha}$ in $(2.9)$ ensures that the function $[z]^{\alpha}$ is single-valued, whereas for generic values of $\alpha$ the holomorphic and anti-holomorphic factors of $[z]^{\alpha}$ taken separately have branch cuts. Bearing in mind that the holomorphic sector is always accompanied by the antiholomorphic one we omit the $\bar{\alpha}$-dependence in the $A$-factor: $A(\alpha, \bar{\alpha}) \rightarrow A(\alpha)$.

Thus, if the normalization in (2.5) is chosen properly, the intertwining operator can be represented in two equivalent forms, either as a formal complex power of the differentiation operator $\mathrm{W}(s, \bar{s})=\left[i \partial_{z}\right]^{2 s+1}$ or as a well defined integral operator

$$
[\mathrm{W}(s, \bar{s}) \Phi](z, \bar{z})=\frac{(-1)^{|s-\bar{s}|}}{\pi} \frac{\Gamma(s+\bar{s}+|s-\bar{s}|+2)}{\Gamma(-s-\bar{s}+|s-\bar{s}|-1)} \int_{\mathbb{C}} \mathrm{d}^{2} x[z-x]^{-2 s-2} \Phi(x, \bar{x}) .
$$


At special points $2 s=n, 2 \bar{s}=\bar{n}, n, \bar{n} \in \mathbb{Z}_{\geq 0}$, the integral operator turns to the differential operator of a finite order $\left(i \partial_{z}\right)^{n+1}\left(i \partial_{\bar{z}}\right)^{\bar{n}+1}$. Let us note that for generic $s$ the holomorphic $\partial_{z}^{2 s+1}$ and anti-holomorphic $\partial_{\bar{z}}^{2 \bar{s}+1}$ parts (see (2.9)) of the intertwiner $\left[i \partial_{z}\right]^{2 s+1}$ taken separately are ill-defined (working with the contour integrals with the kernel $(z-x)^{\alpha}$ one cannot find a translationally invariant measure). However, being taken together, they form a well-defined integral operator.

Formula (2.1) implies that for special values of spins $2 s=n, 2 \bar{s}=\bar{n}$ discussed above an $(n+1)(\bar{n}+1)$-dimensional representation decouples from the general infinite-dimensional case [23]. Indeed, the space of polynomials spanned by $(n+1)(\bar{n}+1)$ basis vectors $z^{k} \bar{z}^{\bar{k}}$, where $k=$ $0,1, \ldots, n$ and $\bar{k}=0,1, \ldots, \bar{n}$, is invariant with respect to the action of the operators $\mathrm{T}^{(s, \bar{s})}(\mathrm{g})$. Instead of working with the separate basis vectors we prefer to deal with a single generating function which contains all of them. The generating function for basis vectors of this finitedimensional representation can be chosen in the following form

$$
[z-x]^{n}=(z-x)^{n}(\bar{z}-\bar{x})^{\bar{n}}
$$

where $x, \bar{x}$ are some auxiliary parameters. Indeed, expanding (2.11) with respect to $x$ and $\bar{x}$ we recover all $(n+1)(\bar{n}+1)$ vectors $z^{k} \bar{z}^{\bar{k}}$, where $k=0,1, \ldots, n$ and $\bar{k}=0,1, \ldots, \bar{n}$.

The decoupling of a finite-dimensional representation and the explicit expression for the generating function (2.11) allow us to give a very natural interpretation to the situation from the point of view of the intertwining operator. Indeed, an immediate consequence of the definition (2.3) is that the null-space of $\mathrm{W}(s, \bar{s})$ - the space annihilated by the operator - is invariant under the action of the operators $\mathrm{T}^{(s, \bar{s})}(\mathrm{g})$. Therefore, if the intertwining operator has a nontrivial nullspace then a sub-representation decouples and the corresponding invariant subspace appears. In the case at hand, when $2 s=n$ and $2 \bar{s}=\bar{n}$, the intertwining operator turns into the differential operator $\partial^{n+1} \bar{\partial}^{\bar{n}+1}$.

Of course this operator annihilates all $(n+1)(\bar{n}+1)$ basis vectors $z^{k} \bar{z}^{\bar{k}}$, where $k=0,1, \ldots, n$ and $\bar{k}=0,1, \ldots, \bar{n}$, but the whole null-space of this operator is too big (it includes all harmonic functions) and we need some additional characterization for the considered finite-dimensional subspace. Relation (2.3) shows that the image of the intertwining operator $\mathrm{W}(-1-s,-1-\bar{s})$ is also invariant under the action of the operators $\mathrm{T}^{(s, \bar{s})}(g)$. Moreover, formula $(2.10)$ in the considered situation

$$
\begin{aligned}
& {[\mathrm{W}(-1-s,-1-\bar{s}) \Phi](z, \bar{z})} \\
& \quad=\frac{(-1)^{|s-\bar{s}|}}{\pi} \frac{\Gamma(-s-\bar{s}+|s-\bar{s}|)}{\Gamma(s+\bar{s}+|s-\bar{s}|+1)} \int_{\mathbb{C}} d^{2} x(z-x)^{2 s}(\bar{z}-\bar{x})^{2 \bar{s}} \Phi(x, \bar{x}),
\end{aligned}
$$

clearly shows that for special values of the spins $2 s=n$ and $2 \bar{s}=\bar{n}$ discussed above the integral in the right-hand side is equal to a polynomial with respect to $z$ and $\bar{z}$, and the image of the operator $\mathrm{W}(-1-s,-1-\bar{s}$ ) (after dropping the numerical factor $\Gamma(-s-\bar{s}+|s-\bar{s}|)$ which diverges at these points) is exactly the needed finite-dimensional subspace. After all we obtain a characterization of our finite-dimensional subspace: it is the intersection of the null-space of the intertwining operator $\mathrm{W}(s, \bar{s})$ and of the image of the operator $\mathrm{W}(-1-s,-1-\bar{s})$ both being properly normalized for special values of the spins $2 s=n$ and $2 \bar{s}=\bar{n}$.

The intertwining operator annihilates the generating function of the finite-dimensional representation (2.11), which can be seen solely from its basic properties. The following calculation suggests this generating function itself. The formal differential operator form of the intertwining operators

$$
\mathrm{W}(s)=\left[i \partial_{z}\right]^{2 s+1}, \quad \mathrm{~W}(-1-s)=\left[i \partial_{z}\right]^{-1-2 s}
$$


formally indicates that $\mathrm{W}(-1-s)$ and $\mathrm{W}(s)$ are inverses to each other,

$$
\mathrm{W}(s) \mathrm{W}(-1-s)=\mathbb{1} .
$$

However, this inversion relation is broken for special values of the spins. Let us rewrite the identity (2.13) taking into account the explicit expression for kernels of the integral operators $\mathrm{W}(-1-s)(2.12)$ and $\mathbb{1}$, which is given by the Dirac delta-function. In this way we find the relation

$$
\left[i \partial_{z}\right]^{2 s+1}[z-x]^{2 s}=(-1)^{-|s-\bar{s}|} \pi \frac{\Gamma(s+\bar{s}+|s-\bar{s}|+1)}{\Gamma(-s-\bar{s}+|s-\bar{s}|)} \delta^{2}(z-x) .
$$

At special points $2 s=n, 2 \bar{s}=\bar{n}$ the gamma-function $\Gamma(-s-\bar{s}+|s-\bar{s}|)$ has poles, and therefore the right-hand side of the latter formula vanishes. So, one obtains

$$
\left[i \partial_{z}\right]^{n+1}[z-x]^{n}=0, \quad n=0,1,2, \ldots,
$$

i.e., the generating function of the finite-dimensional representation coincides with the kernel of the intertwining operator $\mathrm{W}(-1-n / 2)$ after a proper normalization.

Our calculation may seem superfluous since the relation (2.14) is evident per se. However, we presented it here because all its basic steps remain valid after the trigonometric (see Section 3.1) and elliptic deformations (see [16, 17]) of the symmetry algebra. The deformations complicate significantly the intertwining operator and the generating function of finite-dimensional representations such that the deformed analogues of (2.14) are far from being obvious and in the elliptic case they are much more involved [10, 17].

\subsection{The general $\mathrm{SL}(2, \mathbb{C})$-invariant $\mathrm{R}$-operator}

Emergence of the periodic integrable spin chain with $\mathrm{SL}(2, \mathbb{C})$ symmetry in the high energy asymptotics of quantum chromodynamics was discovered in [21, 30, 31]. The detailed consideration of the corresponding formalism was performed in $[12,14]$. In these papers the quantummechanical model of interest has been solved, i.e., the relevant Baxter Q-operator has been constructed and the separation of variables has been implemented. The general R-operator for the $\mathrm{SL}(2, \mathbb{C})$ group has been extensively studied in the first part of [15] as a simplest nontrivial example of the general $\mathrm{SL}(N, \mathbb{C})$-construction. Here we briefly outline main steps in the construction of this R-operator before proceeding to its finite-dimensional reductions.

Firstly we tailor a pair of L-operators out of the Lie algebra generators $\mathrm{E}^{(s)}, \overline{\mathrm{E}}^{(\bar{s})}(2.2)$ and the spectral parameters $u$ and $\bar{u}$ which are assumed to be restricted similar to the representation parameters, $u-\bar{u} \in \mathbb{Z}[14,15]$,

$$
\begin{aligned}
& \mathrm{L}\left(u_{1}, u_{2}\right)=u \cdot \mathbb{1}+\mathrm{E}^{(s)}=\left(\begin{array}{ll}
1 & 0 \\
z & 1
\end{array}\right)\left(\begin{array}{cc}
u_{1} & -\partial \\
0 & u_{2}
\end{array}\right)\left(\begin{array}{cc}
1 & 0 \\
-z & 1
\end{array}\right), \\
& \overline{\mathrm{L}}\left(\bar{u}_{1}, \bar{u}_{2}\right)=\bar{u} \cdot \mathbb{1}+\overline{\mathrm{E}}^{(\bar{s})}=\left(\begin{array}{ll}
1 & 0 \\
\bar{z} & 1
\end{array}\right)\left(\begin{array}{cc}
\bar{u}_{1} & -\bar{\partial} \\
0 & \bar{u}_{2}
\end{array}\right)\left(\begin{array}{cc}
1 & 0 \\
-\bar{z} & 1
\end{array}\right) .
\end{aligned}
$$

Here we use the convenient shorthand notation

$$
u_{1}=u-s-1, \quad u_{2}=u+s, \quad \bar{u}_{1}=\bar{u}-\bar{s}-1, \quad \bar{u}_{2}=\bar{u}+\bar{s} .
$$

Each of the L-operators (2.15), (2.16) respects the RLL-relation with Yang's $4 \times 4$ R-matrix,

$$
\begin{aligned}
& \mathrm{R}_{a b, e f}(u-v) \mathrm{L}_{e c}(u) \mathrm{L}_{f d}(v)=\mathrm{L}_{b f}(v) \mathrm{L}_{a e}(u) \mathrm{R}_{e f, c d}(u-v), \\
& \mathrm{R}_{a b, e f}(\bar{u}-\bar{v}) \overline{\mathrm{L}}_{e c}(\bar{u}) \overline{\mathrm{L}}_{f d}(\bar{v})=\overline{\mathrm{L}}_{b f}(\bar{v}) \overline{\mathrm{L}}_{a e}(\bar{u}) \mathrm{R}_{e f, c d}(\bar{u}-\bar{v}),
\end{aligned}
$$


where $a, b, \ldots=1,2$, and the summation over repeated indices is assumed, $\mathrm{R}_{a b, c d}(u)=u$. $\delta_{a c} \delta_{b d}+\delta_{a d} \delta_{b c}$ (cf. (2.49)). The described relations supplemented by the commutativity condition $[\mathrm{L}(u), \overline{\mathrm{L}}(\bar{v})]=0$ are equivalent to the set of commutation relations for the Lie algebra generators of $\operatorname{SL}(2, \mathbb{C})$.

The L-operators (2.15), (2.16) respect simultaneously another RLL-relation with some general $\mathrm{R}$-operator which intertwines the co-product of L-operators in the pair of quantum spaces

$$
\begin{aligned}
& \mathrm{R}_{12}(u-v, \bar{u}-\bar{v}) \mathrm{L}_{1}\left(u_{1}, u_{2}\right) \mathrm{L}_{2}\left(v_{1}, v_{2}\right)=\mathrm{L}_{1}\left(v_{1}, v_{2}\right) \mathrm{L}_{2}\left(u_{1}, u_{2}\right) \mathrm{R}_{12}(u-v, \bar{u}-\bar{v}), \\
& \mathrm{R}_{12}(u-v, \bar{u}-\bar{v}) \overline{\mathrm{L}}_{1}\left(\bar{u}_{1}, \bar{u}_{2}\right) \overline{\mathrm{L}}_{2}\left(\bar{v}_{1}, \bar{v}_{2}\right)=\overline{\mathrm{L}}_{1}\left(\bar{v}_{1}, \bar{v}_{2}\right) \overline{\mathrm{L}}_{2}\left(\bar{u}_{1}, \bar{u}_{2}\right) \mathrm{R}_{12}(u-v, \bar{u}-\bar{v}),
\end{aligned}
$$

where parameters $u_{1}$ and $u_{2}$ are defined in (2.17), and $v_{1}, v_{2}$ are analogous linear combinations of $v$ and $\ell$,

$$
v_{1}=v-\ell-1, \quad v_{2}=v+\ell, \quad \bar{v}_{1}=\bar{v}-\bar{\ell}-1, \quad \bar{v}_{2}=\bar{v}+\bar{\ell} .
$$

The lower indices of $\mathrm{R}_{12}$ and $\mathrm{L}_{1}, \mathrm{~L}_{2}$ denote quantum spaces on which the operators act nontrivially. The L-operators are multiplied as conventional $2 \times 2$ matrices and the R-operator acts as an identity operator on the auxiliary 2-dimensional spaces of L-operators, but it acts non-trivially on the tensor product of two infinite-dimensional representations: the first representation is specified by the spins $s, \bar{s}$ and it is realized on the functions of variables $z_{1}, \bar{z}_{1}$, the second representation is specified by the spins $\ell, \bar{\ell}$ and it is realized on the functions of variables $z_{2}, \bar{z}_{2}$. In (2.19), (2.20) we drop dependencies of the R-operator on the representation parameters. The full-fledged notation would be $\mathrm{R}(u-v, \bar{u}-\bar{v} \mid s, \bar{s}, \ell, \bar{\ell})$.

Note that the R-operator serves for both L-operators, i.e., it is not just the holomorphic or anti-holomorphic object, as opposed to the L-operators (2.15), (2.16). In the following we frequently omit the dependence of the R-operator (and other intertwining operators) on the anti-holomorphic parameters denoting it $\mathrm{R}(u)$. The $\mathrm{R}$-operator is invariant with respect to the $\mathrm{SL}(2, \mathbb{C})$ group, i.e., it commutes with the co-product of $\mathfrak{s l}(2, \mathbb{C})$ generators

$$
\left[\mathrm{R}_{12}(u, \bar{u}), \mathrm{E}_{1}^{(s)}+\mathrm{E}_{2}^{(\ell)}\right]=0, \quad\left[\mathrm{R}_{12}(u, \bar{u}), \overline{\mathrm{E}}_{1}^{(\bar{s})}+\overline{\mathrm{E}}_{2}^{(\bar{\ell})}\right]=0,
$$

which follows immediately from the RLL-relations (2.19) and (2.20).

Apart from the RLL-relations (2.19), (2.20) the general R-operator satisfies the YBE

$$
\mathrm{R}_{23}(u-v, \bar{u}-\bar{v}) \mathrm{R}_{12}(u, \bar{u}) \mathrm{R}_{23}(v, \bar{v})=\mathrm{R}_{12}(v, \bar{v}) \mathrm{R}_{23}(u, \bar{u}) \mathrm{R}_{12}(u-v, \bar{u}-\bar{v}),
$$

where both sides are endomorphisms on the tensor product of three infinite-dimensional spaces realizing arbitrary principal series representations of $\mathrm{SL}(2, \mathbb{C})$.

In $[14,15]$ an integral operator solution of the intertwining relations (2.19) and (2.20) was found, which solves simultaneously YBE (2.21). The construction naturally gives to this general R-operator several factorized forms related to an integral operator realization of the generators of symmetric group $\mathfrak{S}_{4}$ [15]. Here we do not go into details of this formalism and just indicate the factorization which is appropriate for our current purposes. The R-operator can be represented as a product of four elementary intertwining operators [15]

$$
\mathrm{R}_{12}(u-v, \bar{u}-\bar{v})=\left[z_{12}\right]^{u_{2}-v_{1}}\left[i \partial_{2}\right]^{u_{1}-v_{1}}\left[i \partial_{1}\right]^{u_{2}-v_{2}}\left[z_{12}\right]^{u_{1}-v_{2}},
$$

where we assume the shorthand notation $z_{i j}=z_{i}-z_{j}$ and (2.9). Taking into account (2.8) one can rewrite (2.22) explicitly as an integral operator. The notation (2.9) implies that the Roperator consists of the holomorphic and anti-holomorphic parts which, being taken separately, are ill-defined for generic spectral and representation parameters. The merge of holomorphic and antiholomorphic parts yields a well-defined integral operator. 
Formula (2.22) plays a crucial role in the subsequent discussion. It admits deformations [13] leading to R-operators for the modular double [6] and the elliptic modular double [16].

The expression (2.22) may seem rather unusual. In [15] it was shown that the holomorphic part of the R-operator (2.22), being restricted to the space of polynomials, coincides with the familiar R-operator constructed in $[28,39]$ in the form of the beta-function depending on the "square root" of the Casimir operator. However, the form (2.22) does not demand extra information about the structure of tensor products and corresponding Clebsch-Gordan coefficients. Furthermore, we will show that the integral R-operator (2.22) contains finite-dimensional solutions of the Yang-Baxter relation as well (2.21).

The elementary intertwining operators appearing in (2.22) fulfill the following operator relations

$$
\left[i \partial_{k}\right]^{a}\left[z_{12}\right]^{a+b}\left[i \partial_{k}\right]^{b}=\left[z_{12}\right]^{b}\left[i \partial_{k}\right]^{a+b}\left[z_{12}\right]^{a}, \quad k=1,2 .
$$

These formulae have a remarkable interpretation in terms of the Coxeter relations of the symmetric group $\mathfrak{S}_{4}[13,15]$. Using $(2.23)$ one can easily prove that the R-operator $(2.22)$ respects the YBE (2.21). The operator factors in (2.22) are called intertwiners because they satisfy the equations

$$
\begin{aligned}
& {\left[i \partial_{1}\right]^{u_{2}-u_{1}} \mathrm{~L}_{1}\left(u_{1}, u_{2}\right)=\mathrm{L}_{1}\left(u_{2}, u_{1}\right)\left[i \partial_{1}\right]^{u_{2}-u_{1}},} \\
& {\left[i \partial_{2}\right]^{v_{2}-v_{1}} \mathrm{~L}_{2}\left(v_{1}, v_{2}\right)=\mathrm{L}_{2}\left(v_{2}, v_{1}\right)\left[i \partial_{2}\right]^{v_{2}-v_{1}},} \\
& {\left[z_{12}\right]^{u_{1}-v_{2}} \mathrm{~L}_{1}\left(u_{1}, u_{2}\right) \mathrm{L}_{2}\left(v_{1}, v_{2}\right)=\mathrm{L}_{1}\left(v_{2}, u_{2}\right) \mathrm{L}_{2}\left(v_{1}, u_{1}\right)\left[z_{12}\right]^{u_{1}-v_{2}},}
\end{aligned}
$$

and similar ones with $\mathrm{L}$ substituted by $\overline{\mathrm{L}}$. Here the operators $\left[i \partial_{k}\right]^{a}$ and $\left[z_{12}\right]^{a}$ act on each matrix element of the matrices $\mathrm{L}_{k}$ entrywise, i.e., they should be considered as $2 \times 2$ diagonal matrices proportional to the unit matrix. Moreover, the latter relations fix uniquely (up to a normalization) the elementary intertwining operators. Note that $\left[i \partial_{1}\right]^{u_{2}-u_{1}}=\left[i \partial_{1}\right]^{2 s+1}$ and $\left[i \partial_{2}\right]^{v_{2}-v_{1}}=\left[i \partial_{2}\right]^{2 \ell+1}$ are the intertwining operators of the equivalent representations (2.3) for the first and second spaces, respectively. The equalities (2.24) are identical to the defining relations (2.3) of the intertwining operator W. Applying several times (2.24) and (2.25) one can easily check that the composite R-operator (2.22) obeys the RLL-relations (2.19) and (2.20).

The identities (2.23) are equivalent to the famous star-triangle relation which can be represented in the following three equivalent forms:

1) as an integral identity $[14,41]$

$$
\begin{array}{rl}
\int_{\mathbb{C}} d^{2} & w \frac{1}{[z-w]^{\alpha}[w-x]^{\beta}[w-y]^{\gamma}} \\
& =\frac{A(-\beta)}{A(\alpha-1) A(\gamma-1)} \frac{1}{[z-x]^{1-\gamma}[z-y]^{1-\beta}[y-x]^{1-\alpha}},
\end{array}
$$

provided that the exponents respect the uniqueness conditions

$$
\alpha+\beta+\gamma=\bar{\alpha}+\bar{\beta}+\bar{\gamma}=2
$$

2) as a particular point in the image of the operator $\left[i \partial_{z}\right]^{\alpha-1}$ (with the same restriction on the exponents as before)

$$
\left[i \partial_{z}\right]^{\alpha-1}\left(\frac{1}{[z-x]^{\beta}[z-y]^{\gamma}}\right)=\frac{A(-\beta)}{A(\gamma-1)} \frac{1}{[z-x]^{1-\gamma}[z-y]^{1-\beta}[y-x]^{1-\alpha}}
$$

3) or as a pseudo-differential operators identity [25]

$$
\left[i \partial_{z}\right]^{\alpha} \cdot[z]^{\alpha+\beta} \cdot\left[i \partial_{z}\right]^{\beta}=[z]^{\beta} \cdot\left[i \partial_{z}\right]^{\alpha+\beta} \cdot[z]^{\alpha} .
$$




\subsection{Finite-dimensional reductions of the general R-operator}

Now we reduce the R-operator (2.22) to finite-dimensional representations in its first space. The principal possibility of this reduction is based on the following relation

$$
\left[i \partial_{1}\right]^{u_{2}-u_{1}} \mathbb{R}_{12}\left(u_{1}, u_{2} \mid v_{1}, v_{2}\right)=\mathbb{R}_{12}\left(u_{2}, u_{1} \mid v_{1}, v_{2}\right)\left[i \partial_{1}\right]^{u_{2}-u_{1}},
$$

where we use the $\mathbb{R}$-operator $\mathbb{R}_{12}:=\mathrm{P}_{12} \mathrm{R}_{12}$ with $\mathrm{P}_{12}$ - a permutation operator, $\mathrm{P}_{12} \Psi\left(z_{1}, z_{2}\right)=$ $\Psi\left(z_{2}, z_{1}\right) \mathrm{P}_{12}$. Relation (2.29) can be proved using the identity (2.23) and it shows that both, the null-space of the intertwining operator $\left[i \partial_{1}\right]^{2 s+1}$ and the image of the intertwining operator $\left[i \partial_{1}\right]^{-2 s-1}$, are mapped onto themselves by our R-matrix $\mathbb{R}_{12}$. Therefore, if we find invariant finite-dimensional subspaces of the latter spaces they will be invariant with respect to the action of R-operator itself.

We take the function $\left[z_{13}\right]^{2 s} \Phi\left(z_{2}, \bar{z}_{2}\right)$, where $2 s=u_{2}-u_{1}-1$ and $\Phi\left(z_{2}, \bar{z}_{2}\right)$ is an arbitrary function, and act upon it by the R-operator. We break down the calculation to several steps according to the factorized form (2.22) of the R-operator. At the end of calculation we choose $2 s=n, 2 \bar{s}=\bar{n}$ with $n, \bar{n} \in \mathbb{Z}_{\geq 0}$ such that $\left[z_{13}\right]^{2 s}$ turns into the generating function of the finitedimensional representation in the first space (2.11) with an auxiliary parameter $z_{3}$. However, for a while we assume the spin $s$ to be generic.

Using formula (2.27) we implement the first step.

We act by the first two factors $\left[i \partial_{1}\right]^{u_{2}-v_{2}}\left[z_{12}\right]^{u_{1}-v_{2}}$ of the R-operator (2.22) and find

$$
\begin{aligned}
& {\left[i \partial_{1}\right]^{u_{2}-v_{2}}\left[z_{12}\right]^{u_{1}-v_{2}}\left[z_{13}\right]^{2 s} \Phi\left(z_{2}, \bar{z}_{2}\right)} \\
& \quad=\frac{A\left(u_{1}-v_{2}\right)}{A\left(u_{1}-u_{2}\right)} \cdot\left[z_{12}\right]^{u_{1}-u_{2}}\left[z_{13}\right]^{v_{2}-u_{1}-1}\left[z_{23}\right]^{u_{2}-v_{2}} \Phi\left(z_{2}, \bar{z}_{2}\right) .
\end{aligned}
$$

In order to apply the third factor $\left[i \partial_{2}\right]^{u_{1}-v_{1}}$ of the R-operator (2.22) we resort to the relation

$$
\begin{aligned}
& {\left[i \partial_{2}\right]^{u_{1}-v_{1}}\left[z_{12}\right]^{u_{1}-u_{2}}\left[z_{23}\right]^{u_{2}-v_{2}} \Phi\left(z_{2}, \bar{z}_{2}\right)} \\
& \quad=\frac{A\left(u_{1}-v_{1}\right)}{A\left(u_{2}-u_{1}-1\right)} \cdot\left[i \partial_{1}\right]^{u_{2}-u_{1}-1}\left[z_{12}\right]^{v_{1}-u_{1}-1}\left[z_{13}\right]^{u_{2}-v_{2}} \Phi\left(z_{1}, \bar{z}_{1}\right)
\end{aligned}
$$

which follows immediately from the integral representation $(2.8)$ for $\left[i \partial_{z}\right]^{\alpha}$. A merit of the previous formula is that we traded the integral operator $\left[i \partial_{2}\right]^{u_{1}-v_{1}}$ for $\left[i \partial_{1}\right]^{2 s}$, which becomes just a differential operator for $2 s=n$ and $2 \bar{s}=\bar{n}$. Incorporating into the latter formula the inert factors from (2.30) and the last factor $\left[z_{12}\right]^{u_{2}-v_{1}}$ of the R-operator (2.22), we find

$$
\begin{gathered}
\mathrm{R}_{12}\left(u_{1}, u_{2} \mid v_{1}, v_{2}\right)\left[z_{13}\right]^{u_{2}-u_{1}-1} \Phi\left(z_{2}, \bar{z}_{2}\right)=\frac{A\left(u_{1}-v_{2}\right)}{A\left(u_{1}-u_{2}\right)} \frac{A\left(u_{1}-v_{1}\right)}{A\left(u_{2}-u_{1}-1\right)} \\
\quad \times\left[z_{12}\right]^{u_{2}-v_{1}}\left[z_{13}\right]^{v_{2}-u_{1}-1}\left[i \partial_{1}\right]^{u_{2}-u_{1}-1}\left[z_{12}\right]^{v_{1}-u_{1}-1}\left[z_{13}\right]^{u_{2}-v_{2}} \Phi\left(z_{1}, \bar{z}_{1}\right) .
\end{gathered}
$$

In order to polish the latter formula we denote $z_{3}=x$ like in (2.11) and rewrite (2.31) in terms of the representation parameters. Also we prefer to replace the R-operator by $\mathbb{R}_{12}=\mathrm{P}_{12} \mathrm{R}_{12}$.

Thus the general R-operator for the $\mathrm{SL}(2, \mathbb{C})$ group acting in the tensor product of two infinite-dimensional representation spaces with spins $s, \bar{s}$ and $\ell, \bar{\ell}$ can be reduced to a finitedimensional subspace in the first space if $2 s=n, 2 \bar{s}=\bar{n}\left(n, \bar{n} \in \mathbb{Z}_{\geq 0}\right)$. We have the following formula

$$
\begin{aligned}
\mathbb{R}_{12}\left(u \mid \frac{n}{2}, \frac{\bar{n}}{2}, \ell, \bar{\ell}\right)\left[z_{1}-x\right]^{n} \Phi\left(z_{2}, \bar{z}_{2}\right) \\
\quad=c \cdot\left[z_{2}-x\right]^{-u+\frac{n}{2}+\ell}\left[z_{12}\right]^{u+\frac{n}{2}+\ell+1}\left[\partial_{z_{2}}\right]^{n}\left[z_{12}\right]^{-u+\frac{n}{2}-\ell-1}\left[z_{2}-x\right]^{u+\frac{n}{2}-\ell} \Phi\left(z_{2}, \bar{z}_{2}\right),
\end{aligned}
$$


where the normalization factor is

$$
c=(-1)^{n+\bar{n}} \frac{A\left(u-\frac{n}{2}+\ell\right)}{A\left(-u+\frac{n}{2}+\ell\right)} .
$$

The latter formula gives a number of solutions of the YBE (2.21) which are endomorphisms on the tensor product of an $(n+1)(\bar{n}+1)$-dimensional and an infinite-dimensional spaces.

We consider formula (2.32) as one of the main results of this paper. It gives a concise expression for known higher spin R-operators. They are "mixed" objects in a sense that they are defined on the tensor product of finite-dimensional and infinite-dimensional representations. In addition they can be considered as generalizations of the L-operators from the fundamental to arbitrary finite-dimensional representations. Moreover, the formula (2.32) produces all such solutions of the YBE related to the principal series representation. Its analogue for the modular double is derived in Section 3.3 and the elliptic modular double case is considered in [10].

In order to get accustomed to the reduction formula (2.32) let us consider a simple example. One can easily recover the holomorphic L-operator $(2.15)$ substituting $(n, \bar{n})=(1,0)$ in $(2.32)$ and choosing the basis in the space $\mathbb{C}^{2}$ of the fundamental representation as $\mathbf{e}_{1}=-z_{1}, \mathbf{e}_{2}=1$. Then

$$
\begin{aligned}
& \mathbb{R}_{12}\left(u-\frac{1}{2} \mid \frac{1}{2}, \ell\right) \mathbf{e}_{1}=c \cdot\left[\mathbf{e}_{1}\left(z_{2} \partial_{2}-\ell+u\right)+\mathbf{e}_{2}\left(z_{2}^{2} \partial_{2}-2 \ell z_{2}\right)\right], \\
& \mathbb{R}_{12}\left(u-\frac{1}{2} \mid \frac{1}{2}, \ell\right) \mathbf{e}_{2}=c \cdot\left[\mathbf{e}_{1}\left(-\partial_{2}\right)+\mathbf{e}_{2}\left(u+\ell-z_{2} \partial_{2}\right)\right] .
\end{aligned}
$$

Consequently the restriction of $\mathbb{R}_{12}\left(u-\frac{1}{2} \mid \frac{1}{2}, \ell\right)$ to $\mathbb{C}^{2}$ in the first factor takes the matrix form

$$
\mathrm{L}(u)=\left(\begin{array}{cc}
u-\ell+z \partial & -\partial \\
z^{2} \partial-2 \ell z & u+\ell-z \partial
\end{array}\right)
$$

and coincides with the holomorphic L-operator (2.15). Analogously taking $(n, \bar{n})=(0,1)$ we recover the anti-holomorphic $\overline{\mathrm{L}}$-operator (2.16).

Besides the L-operator, the formula (2.32) reproduces all its higher-spin generalizations. Simultaneously, it produces R-matrices described by plain finite-dimensional matrices in both spaces. Indeed, substituting in (2.32) the generating function (2.11) of the finite-dimensional $(m+1)(\bar{m}+1)$-dimensional representation in the second space, we find a solution of the YBE (2.21) for the spins $\frac{n}{2}, \frac{\bar{n}}{2}$ and $\frac{m}{2}, \frac{\bar{m}}{2}$ in the first and second spaces, respectively,

$$
\begin{aligned}
& \mathbb{R}_{12}\left(u \mid \frac{n}{2}, \frac{\bar{n}}{2}, \frac{m}{2}, \frac{\bar{m}}{2}\right)\left[z_{1}-x\right]^{n}\left[z_{2}-y\right]^{m} \\
& \quad=c \cdot\left[z_{2}-x\right]^{-u+\frac{n}{2}+\ell}\left[z_{12}\right]^{u+\frac{n}{2}+\frac{m}{2}+1}\left[\partial_{z_{2}}\right]^{n}\left[z_{12}\right]^{-u+\frac{n}{2}-\frac{m}{2}-1}\left[z_{2}-x\right]^{u+\frac{n}{2}-\frac{m}{2}}\left[z_{2}-y\right]^{m} .
\end{aligned}
$$

Expanding both sides of this relation in auxiliary parameters $x, \bar{x}, y, \bar{y}$ one can rewrite it in a form of a square matrix with $(n+1)(\bar{n}+1)(m+1)(\bar{m}+1)$ rows (or columns). The compact formula (2.36) produces all its entries. In particular, taking the fundamental representation in both spaces $n=m=1, \bar{n}=\bar{m}=0$ we reproduce Yang's R-matrix (cf. (2.49)).

\subsection{Verma module reduction}

In this section we slightly digress from the discussion of the group $\operatorname{SL}(2, \mathbb{C})$ and outline how $\mathfrak{s l}_{2}$ symmetric finite-dimensional solutions of the YBE arise from the infinite-dimensional ones. Similar to the previous considerations this approach yields a concise expression for finitedimensional solutions that may find various applications. Since the corresponding calculations are essentially based on ideas explained above we will limit ourselves to the statement of the results.

Although the $\mathfrak{s l}_{2}$ algebra is "a half" of the Lie algebra of the group $\operatorname{SL}(2, \mathbb{C})$, it requires a special treatment. We deal with a functional representation of the $\mathfrak{s l}_{2}$-algebra in the space 
of polynomials of one complex variable $\mathbb{C}[z]$. Fixing a generic complex number $s \in \mathbb{C}$ and representing the algebra generators by the first order differential operators given in (2.2) we endow $\mathbb{C}[z]$ with a structure of the Verma module. For generic value of $s$ the module is an infinite-dimensional space with the basis $\left\{1, z, z^{2}, \ldots\right\}$ and there are no invariant subspaces, i.e., the representation is irreducible. Invariant subspaces arise for the discrete set of spin values $2 s=n, n \in \mathbb{Z}_{\geq 0}$. The corresponding $(n+1)$-dimensional representation is irreducible and it is realized on the submodule with the basis $\left\{1, z, \ldots, z^{n}\right\}$.

Since the $\mathfrak{s l}_{2}$ generators are holomorphic, we have a single holomorphic L-operator given in (2.15). Now only the holomorphic spectral parameter $u$ is present. The general R-operator $\mathrm{R}(u \mid s, \ell)$ is defined on the tensor product of two Verma modules with the spins $s$ and $\ell$. It has to satisfy holomorphic analogues of the RLL-relation (2.19) and of the YBE (2.21).

The general R-operator $(2.22)$ for $\operatorname{SL}(2, \mathbb{C})$ group is well defined due to its non-analyticity, in other words, due to the presence of holomorphic and antiholomorphic parts. We cannot get the general R-operator for $\mathfrak{s l}_{2}$ (which has to be holomorphic) by crossing out the anti-holomorphic part of (2.22). Anyway, the holomorphic RLL-relation (2.19) can be solved [15] in terms of a well-defined operator on $\mathbb{C}\left[z_{1}\right] \otimes \mathbb{C}\left[z_{2}\right]$ which takes the following factorized form,

$$
\mathrm{R}_{12}(u \mid s, \ell)=\frac{\Gamma\left(z_{21} \partial_{2}-2 s\right)}{\Gamma\left(z_{21} \partial_{2}-u-s-\ell\right)} \frac{\Gamma\left(z_{12} \partial_{1}+u-s-\ell\right)}{\Gamma\left(z_{12} \partial_{1}-2 s\right)},
$$

where ratios of the operator-valued gamma functions are defined with the help of the integral representation for Euler's beta-function

$$
\frac{\Gamma\left(z_{12} \partial_{1}+a\right)}{\Gamma\left(z_{12} \partial_{1}+b\right)} \Phi\left(z_{1}, z_{2}\right):=\frac{1}{\Gamma(b-a)} \int_{0}^{1} d \alpha \alpha^{a-1}(1-\alpha)^{b-a-1} \Phi\left(\alpha z_{1}+(1-\alpha) z_{2}, z_{2}\right) .
$$

This R-operator satisfies the holomorphic analogue of YBE (2.21) as well. As we remarked in Section 2.2 the operator $(2.37)$ coincides with the one found in $[28,39]$ in the early days of the quantum inverse scattering method in spite of the fact that they look completely different.

For $2 s=n, n \in \mathbb{Z}_{\geq 0}$, the general R-operator (2.37) can be restricted to an $(n+1)$-dimensional representation in the first space. Taking into account permutation of the pair of tensor factors, $\mathbb{R}_{12}=\mathrm{P}_{12} \mathrm{R}_{12}$, one can show that the restricted $\mathrm{R}$-operator acquires a concise form

$$
\begin{aligned}
& \mathbb{R}_{12}\left(u \mid \frac{n}{2}, \ell\right)\left(z_{1}-x\right)^{n} \Phi\left(z_{2}\right) \\
& \quad=c \cdot\left(z_{2}-x\right)^{-u+\frac{n}{2}+\ell} z_{12}^{u+\frac{n}{2}+\ell+1} \partial_{z_{2}}^{n} z_{12}^{-u+\frac{n}{2}-\ell-1}\left(z_{2}-x\right)^{u+\frac{n}{2}-\ell} \Phi\left(z_{2}\right),
\end{aligned}
$$

where the normalization factor is

$$
c=(-1)^{n+1} \frac{\Gamma\left(-\ell-\frac{n}{2}-u\right)}{\Gamma\left(-\ell+\frac{n}{2}-u\right)} .
$$

Formula $(2.38)$ is completely analogous to the $\mathrm{SL}(2, \mathbb{C})$ reduction formula (2.32). Expanding both sides of (2.38) with respect to an auxiliary parameter $x$ one recovers an $(n+1) \times(n+1)$ matrix whose entries are the $n$-th order differential operators with polynomial coefficients in spectral parameter $u$ of degree $n$ (or lower).

In [8] the Lax operator has been recovered from the general R-operator by means of a quite bulky calculation. Formula (2.38) provides considerable simplification of that result generalizing it to the higher-spin analogues of the rational Lax operator.

In order to illustrate the power of the formula (2.38) we present below the R-operator for the spin 1 representation in the first space. In the basis $\mathbf{e}_{1}=1, \mathbf{e}_{2}=z_{1}, \mathbf{e}_{3}=z_{1}^{2}$ of the 3 -dimensional space, the $\mathbb{R}(u \mid 1, \ell)$-operator takes the matrix form (we change notation $z_{2} \rightarrow z$ )

$$
\left(\begin{array}{ccc}
(u+\ell)(u+\ell+1)-2(u+\ell) z \partial+z^{2} \partial^{2} & 2 \ell(u+\ell) z-(u+3 \ell-1) z^{2} \partial+z^{3} \partial^{2} & 2 \ell(2 \ell-1) z^{2}+2(1-2 \ell) z^{3} \partial+z^{4} \partial^{2} \\
2(u+\ell) \partial-2 z \partial^{2} & (u+\ell)(u-\ell+1)+2(2 \ell-1) z \partial-2 z^{2} \partial^{2} & 4 \ell(u-\ell+1) z-2(u-3 \ell+2) z^{2} \partial-2 z^{3} \partial^{2} \\
\partial^{2} & (u-\ell+1) \partial+z \partial^{2} & (u-\ell)(u-\ell+1)+2(u-\ell+1) z \partial+z^{2} \partial^{2}
\end{array}\right) .
$$


Conventional methods demand laborious calculations to reproduce this complicated matrix. In our case the result follows immediately from the formula (2.38). An explicit matrix factorization formula for the operator $\mathbb{R}_{12}\left(u \mid \frac{n}{2}, \ell\right)$ (2.38) generalizing factorization of the L-operator (2.15) was derived in the followup paper [7]. E.g., the $\mathbb{R}(u \mid 1, \ell)$-operator given above factorizes to a product of five more elementary $3 \times 3$ matrices: two lower-triangular, two diagonal and one upper-triangular matrix.

\subsection{Fusion, symbols and the Jordan-Schwinger representation}

The standard procedure for constructing finite-dimensional higher-spin R-operators out of the fundamental one is the fusion procedure [28, 29]. Firstly, we remind how it works in the case of the symmetry algebra $\mathfrak{s l}_{2}$ using a formulation convenient for us. Then in the next section we straightforwardly extend it to the case of the $\mathrm{SL}(2, \mathbb{C})$ group and show that the reduction formula (2.32) is in line with the fusion construction.

For the rank one symmetry algebras underlying an integrable system the recipe of [28, 29] looks as follows. One forms an inhomogeneous monodromy matrix $\mathrm{T}_{i_{1} \ldots i_{n}}^{j_{1} \ldots j_{n}}$ out of L-operators $\mathrm{L}_{i}^{j}$ multiplying them as operators in quantum space and taking tensor products of the auxiliary space $\mathbb{C}^{2}$, and then symmetrizes the monodromy matrix over the spinor indices. The parameters of inhomogeneity have to be adjusted in a proper way. The result $\mathrm{T}_{\left(i_{1} \ldots i_{n}\right)}^{\left(j_{1} \ldots j_{n}\right)}$ is an R-operator which has a higher-spin auxiliary space and solves the YBE. Thus constructing higher-spin R-operators one has to deal with $\operatorname{Sym}\left(\mathbb{C}^{2}\right)^{\otimes n}$ which is a space of symmetric tensors with a number of spinor indices $\Psi_{\left(i_{1} \ldots i_{n}\right)}$. The usual matrix-like action of operators has the form

$$
[\mathrm{T} \Psi]_{\left(i_{1} \ldots i_{n}\right)}=\mathrm{T}_{\left(i_{1} \ldots i_{n}\right)}^{\left(j_{1} \ldots j_{n}\right)} \Psi_{\left(j_{1} \ldots j_{n}\right)}
$$

where the summation over repeated indices is assumed. We prefer not to deal with a multitude of spinor indices. Instead we introduce auxiliary spinors $\lambda=\left(\lambda_{1}, \lambda_{2}\right), \mu=\left(\mu_{1}, \mu_{2}\right)$ and contract them with the tensors

$$
\lambda_{i_{1}} \cdots \lambda_{i_{n}} \Psi_{i_{1} \ldots i_{n}}=\Psi(\lambda), \quad \lambda_{i_{1}} \cdots \lambda_{i_{n}} \mathrm{~T}_{i_{1} \ldots i_{n}}^{j_{1} \ldots j_{n}} \mu_{j_{1}} \cdots \mu_{j_{n}}=\mathrm{T}(\lambda \mid \mu)
$$

Thus the symmetization over spinor indices is taken into account automatically. Henceforth, in place of the tensors we work with the corresponding generating functions which are homogeneous polynomials of degree $n$ of two variables

$$
\Psi(\lambda)=\Psi\left(\lambda_{1}, \lambda_{2}\right), \quad \Psi\left(\alpha \lambda_{1}, \alpha \lambda_{2}\right)=\alpha^{n} \Psi\left(\lambda_{1}, \lambda_{2}\right) .
$$

$\mathrm{T}(\lambda \mid \mu)$ is usually called the symbol of the operator. In this way formula (2.39) acquires a rather compact form

$$
[\mathrm{T} \Psi](\lambda)=\left.\frac{1}{n !} \mathrm{T}\left(\lambda \mid \partial_{\mu}\right) \Psi(\mu)\right|_{\mu=0} .
$$

Note that, in fact, we do not need to take $\mu=0$ in (2.42). The $\mu$ variable disappears automatically since $\mathrm{T}(\lambda \mid \mu)$ and $\Psi(\mu)$ have equal homogeneity degrees.

In order to illustrate the merits of auxiliary spinors let us apply them to the text-book example of the quantum-mechanical system of spin $\frac{n}{2}$, i.e., consider the symmetry group $\mathrm{SU}(2)$ and the generators $\vec{J}$ of the Lie algebra $s u_{2}$ in the representation of spin $\frac{n}{2}$. In the spin $\frac{1}{2}$ representation the generators act on the space $\mathbb{C}^{2}$ and they are given by the Pauli matrices $\frac{\vec{\sigma}}{2}$, so that

$$
[\vec{J} \Psi]_{i}=\frac{1}{2} \vec{\sigma}_{i}^{j} \Psi_{j}, \quad \vec{J}_{i}^{j}=\frac{1}{2} \vec{\sigma}_{i}^{j}
$$


Here the lower indices enumerate the rows and the upper indices - the columns. Taking the tensor product of $n$ spin $\frac{1}{2}$ representations we obtain the generators on the space $\left(\mathbb{C}^{2}\right)^{\otimes n}$,

$$
\vec{J}_{i_{1} \ldots i_{n}}^{j_{1} \ldots j_{n}}=\frac{1}{2} \vec{\sigma}_{i_{1}}^{j_{1}} \delta_{i_{2}}^{j_{2}} \cdots \delta_{i_{n}}^{j_{n}}+\cdots+\frac{1}{2} \delta_{i_{1}}^{j_{1}} \cdots \delta_{i_{n-1}}^{j_{n-1}} \vec{\sigma}_{i_{n}}^{j_{n}} .
$$

In order to single out in the tensor product an irreducible maximal spin representation we symmetrize over spinor indices yielding the representation of spin $\frac{n}{2}$,

$$
[\vec{J} \Psi]_{\left(i_{1} \ldots i_{n}\right)}=\frac{1}{2} \vec{\sigma}_{i_{1}}^{j} \Psi_{\left(j i_{2} \ldots i_{n}\right)}+\cdots+\frac{1}{2} \vec{\sigma}_{i_{n}}^{j} \Psi_{\left(i_{1} \ldots i_{n-1} j\right)} .
$$

Further we introduce a pair of auxiliary spinors and find the symbol $\vec{J}(\lambda, \mu)$ of the operator $\vec{J}(2.43)$ converting formula (2.43) to

$$
\begin{aligned}
& \vec{J}(\lambda \mid \mu)=\lambda_{i_{1}} \cdots \lambda_{i_{n}} \vec{J}_{i_{1} \ldots i_{n}}^{j_{1} \ldots j_{n}} \mu_{j_{1}} \cdots \mu_{j_{n}}=\frac{n}{2}\langle\lambda \mid \mu\rangle^{n-1}\langle\lambda|\vec{\sigma}| \mu\rangle, \\
& \left\langle\lambda\left|=\left(\lambda_{1}, \lambda_{2}\right), \quad\right| \mu\right\rangle=\left(\begin{array}{c}
\mu_{1} \\
\mu_{2}
\end{array}\right),
\end{aligned}
$$

where $\langle\lambda \mid \mu\rangle=\lambda_{1} \mu_{1}+\lambda_{2} \mu_{2}$ and $\langle\lambda|\vec{\sigma}| \mu\rangle=\lambda_{i} \vec{\sigma}_{i}^{j} \mu_{j}$ are symbols of the identity operator and Pauli matrices, respectively. In view of (2.42), (2.45), formula (2.44) acquires the indexless form

$$
[\vec{J} \Psi]\left(\lambda_{1}, \lambda_{2}\right)=\left.\frac{1}{n !} \frac{n}{2}\left\langle\lambda \mid \partial_{\mu}\right\rangle^{n-1}\left\langle\lambda|\vec{\sigma}| \partial_{\mu}\right\rangle \Psi(\mu)\right|_{\mu=0} .
$$

Consequently, instead of tensors and finite-dimensional operators we deal with their symbols and generating functions. Note that due to the homogeneity of $\Psi(2.41),\langle\lambda \mid \mu\rangle^{n}$ is a symbol of the identity operator defined on the tensor product of $n$ spaces

$$
\left.\frac{1}{n !}\left\langle\lambda \mid \partial_{\mu}\right\rangle^{n} \Psi(\mu)\right|_{\mu=0}=\left.\frac{1}{n !} \partial_{\alpha}^{n} e^{\alpha\left\langle\lambda \mid \partial_{\mu}\right\rangle} \Psi(\mu)\right|_{\mu=0, \alpha=0}=\left.\frac{1}{n !} \partial_{\alpha}^{n} \Psi(\alpha \lambda)\right|_{\alpha=0}=\Psi(\lambda) .
$$

Then taking into account that

$$
\frac{n}{2}\langle\lambda \mid \mu\rangle^{n-1}\langle\lambda|\vec{\sigma}| \mu\rangle=\frac{1}{2}\left\langle\lambda|\vec{\sigma}| \partial_{\lambda}\right\rangle\langle\lambda \mid \mu\rangle^{n}
$$

we obtain an alternative expression for $\vec{J}$,

$$
[\vec{J} \Psi]\left(\lambda_{1}, \lambda_{2}\right)=\left.\frac{1}{2}\left\langle\lambda|\vec{\sigma}| \partial_{\lambda}\right\rangle \frac{1}{n !}\left\langle\lambda \mid \partial_{\mu}\right\rangle^{n} \Psi(\mu)\right|_{\mu=0}=\frac{1}{2}\left\langle\lambda|\vec{\sigma}| \partial_{\lambda}\right\rangle \Psi(\lambda) .
$$

Thus we have realized the Lie algebra generators $\vec{J}$ as differential operators on the space of homogeneous polynomials of two variables (forming a projective space)

$$
J_{ \pm}=\frac{1}{2}\left\langle\lambda\left|\sigma_{1} \pm i \sigma_{2}\right| \partial_{\lambda}\right\rangle=\left\langle\lambda\left|\sigma_{ \pm}\right| \partial_{\lambda}\right\rangle, \quad J_{3}=\frac{1}{2}\left\langle\lambda\left|\sigma_{3}\right| \partial_{\lambda}\right\rangle
$$

or, more explicitly,

$$
J_{+}=\lambda_{1} \partial_{\lambda_{2}}, \quad J_{-}=\lambda_{2} \partial_{\lambda_{1}}, \quad J_{3}=\frac{1}{2}\left(\lambda_{1} \partial_{\lambda_{1}}-\lambda_{2} \partial_{\lambda_{2}}\right)
$$

This realization of the generators is known as the Jordan-Schwinger representation. We can choose the homogeneous function $\left(\lambda_{1}+x \lambda_{2}\right)^{n}$ (see (2.41)) as a generating function of the $(n+1)$ dimensional representation with an auxiliary parameter $x$.

One can easily proceed from the projective space to the space of polynomials of one complex variable. Indeed, due to the homogeneity

$$
\Psi\left(\lambda_{1}, \lambda_{2}\right)=\lambda_{2}^{n} \Psi\left(\frac{\lambda_{1}}{\lambda_{2}}, 1\right)=\lambda_{1}^{n} \Psi\left(1, \frac{\lambda_{2}}{\lambda_{1}}\right)
$$


all information about $\Psi\left(\lambda_{1}, \lambda_{2}\right)$ is encoded in a function of the ratio $\frac{\lambda_{1}}{\lambda_{2}}$ alone. In order to make contact with the holomorphic set of the $\mathfrak{s l}(2, \mathbb{C})$ generators $(2.2)$ we choose $\lambda_{1}=-z, \lambda_{2}=1$ and rewrite the generators (2.47) in terms of the variable $z$

$$
J_{+}=z^{2} \partial-n z, \quad J_{-}=-\partial, \quad J_{3}=z \partial-\frac{n}{2} .
$$

Furthermore, the generating function of the Jordan-Schwinger representation turns into the generating function of one variable $(x-z)^{n}$ (cf. (2.11), recall (2.38)).

Before proceeding to the fusion procedure for the $\mathrm{SL}(2, \mathbb{C})$ group, we remind construction of the L-operator. We build the L-operator with a finite-dimensional local quantum space starting from the Yang R-matrix. The latter acts on the tensor product of two spin- $\frac{1}{2}$ representations

$$
\mathrm{R}(u)=u+\frac{1}{2}(\mathbb{1}+\vec{\sigma} \otimes \vec{\sigma})=\left(\begin{array}{cc}
u+\frac{1}{2}+\frac{1}{2} \sigma_{3} & \sigma_{-} \\
\sigma_{+} & u+\frac{1}{2}-\frac{1}{2} \sigma_{3}
\end{array}\right) .
$$

Following the recipe from $[28,29]$ we form the product of the Yang R-matrices

$$
\mathrm{R}_{\left(i_{1} \ldots i_{n}\right)}^{\left(j_{1} \ldots j_{n}\right)}(u)=\operatorname{Sym}_{i_{1}}^{j_{1}}(u) \mathrm{R}_{i_{2}}^{j_{2}}(u-1) \cdots \mathrm{R}_{i_{n}}^{j_{n}}(u-n+1),
$$

where the indices refer to the first space in (2.49),

$$
\mathrm{R}_{i}^{j}(u)=\left(u+\frac{1}{2}\right) \delta_{i}^{j}+\frac{1}{2} \vec{\sigma}_{i}^{j} \vec{\sigma},
$$

and Sym implies symmetrization with respect to $\left(i_{1} \ldots i_{n}\right)$ and $\left(j_{1} \ldots j_{n}\right)$. In such a way one obtains an operator acting on the space of symmetric rank $n$ tensors, i.e., on the space of spin $\frac{n}{2}$ representation, and on the two-dimensional auxiliary space where the $\vec{\sigma}$-matrices are acting. According to $[28,29]$ it respects the Yang-Baxter relations. Now we calculate the symbol of (2.50) with respect to the quantum space

$$
\begin{aligned}
\mathrm{R}(u \mid \lambda, \mu) & =\lambda_{i_{1}} \cdots \lambda_{i_{n}} \mathrm{R}_{i_{1} \ldots i_{n}}^{j_{1} \ldots j_{n}}(u) \mu_{j_{1}} \cdots \mu_{j_{n}} \\
& =\langle\lambda|\mathrm{R}(u)| \mu\rangle\langle\lambda|\mathrm{R}(u-1)| \mu\rangle \cdots\langle\lambda|\mathrm{R}(u-n+1)| \mu\rangle,
\end{aligned}
$$

i.e., it is still an operator in the auxiliary space. Henceforth for the sake of brevity we refer to it as a symbol of the R-matrix. The derived symbol $\mathrm{R}(u \mid \lambda, \mu)$ factorizes to a product of Yang's R-matrix symbols $\langle\lambda|\mathrm{R}(u)| \mu\rangle=\lambda_{i} \mathrm{R}_{i}^{j}(u) \mu_{j}$,

$$
\langle\lambda|\mathrm{R}(u)| \mu\rangle=\langle\lambda \mid \mu\rangle\left(u+\frac{1}{2}+\frac{1}{2} \vec{n} \vec{\sigma}\right)=\left(\begin{array}{cc}
(u+1) \lambda_{1} \mu_{1}+u \lambda_{2} \mu_{2} & \lambda_{2} \mu_{1} \\
\lambda_{1} \mu_{2} & u \lambda_{1} \mu_{1}+(u+1) \lambda_{2} \mu_{2}
\end{array}\right),
$$

where we introduced the unit vector $\vec{n}=\frac{\langle\lambda|\vec{\sigma}| \mu\rangle}{\langle\lambda \mid \mu\rangle}, \vec{n} \cdot \vec{n}=1$. The product of such matrices is easy to calculate and we obtain

$$
\begin{aligned}
& \mathrm{R}(u \mid \lambda, \mu)=u(u-1) \cdots(u-n+1) \\
& \times\left(\begin{array}{cc}
\left(u+1-\frac{n}{2}\right)\langle\lambda \mid \mu\rangle^{n}+\frac{n}{2}\langle\lambda \mid \mu\rangle^{n-1}\left(\lambda_{1} \mu_{1}-\lambda_{2} \mu_{2}\right) & n\langle\lambda \mid \mu\rangle^{n-1} \lambda_{2} \mu_{1} \\
n\langle\lambda \mid \mu\rangle^{n-1} \lambda_{1} \mu_{2} & \left(u+1-\frac{n}{2}\right)\langle\lambda \mid \mu\rangle^{n}-\frac{n}{2}\langle\lambda \mid \mu\rangle^{n-1}\left(\lambda_{1} \mu_{1}-\lambda_{2} \mu_{2}\right)
\end{array}\right) .
\end{aligned}
$$

In compact notation this formula takes the form

$$
\begin{aligned}
\mathrm{R}(u \mid \lambda, \mu) & =\langle\lambda \mid \mu\rangle^{n}\left(u+\frac{1}{2}+\frac{1}{2} \vec{n} \vec{\sigma}\right)\left(u-\frac{1}{2}+\frac{1}{2} \vec{n} \vec{\sigma}\right) \cdots\left(u-n+\frac{3}{2}+\frac{1}{2} \vec{n} \vec{\sigma}\right) \\
& =u(u-1) \cdots(u-n+1)\langle\lambda \mid \mu\rangle^{n}\left(u+1-\frac{n}{2}+\frac{n}{2} \vec{n} \vec{\sigma}\right)
\end{aligned}
$$

and it can be easily proven by induction using the identity $(\vec{n} \vec{\sigma})^{2}=\mathbb{1}$. 
Up to the inessential normalization factor $r_{n}(u)=u(u-1) \cdots(u-n+1)$ and the shift of the spectral parameter $u \rightarrow u-1+\frac{n}{2}$, we obtain the following symbol (see (2.45))

$$
\begin{aligned}
\mathrm{L}(u \mid \lambda, \mu) & =r_{n}^{-1}(u) \mathrm{R}\left(u-1+\frac{n}{2} \mid \lambda, \mu\right) \\
& =u\langle\lambda \mid \mu\rangle^{n}+\frac{n}{2}\langle\lambda \mid \mu\rangle^{n-1}\langle\lambda|\vec{\sigma}| \mu\rangle \vec{\sigma}=u\langle\lambda \mid \mu\rangle^{n}+\vec{J}(\lambda, \mu) \vec{\sigma}
\end{aligned}
$$

for the higher-spin R-operator which acts on the tensor product of the spin $\frac{n}{2}$ and spin $\frac{1}{2}$ representations. Such an R-operator is usually called the Lax operator with an $(n+1)$-dimensional local quantum space. Let us emphasize once more that (2.54) is a symbol of the Lax operator solely with respect to the local quantum space, but it is a matrix in the 2-dimensional auxiliary space. In order to avoid misunderstandings we showed in (2.53) its explicit matrix form. The expression $\langle\lambda \mid \mu\rangle^{n}$ is a symbol of the unit operator and $\vec{J}(\lambda, \mu)$ is a symbol of the Lie algebra generators. Hence the fusion procedure yields the familiar Lax operator,

$$
\mathrm{L}(u)=u \mathbb{1}+\vec{J} \vec{\sigma}=\left(\begin{array}{cc}
u+J_{3} & J_{-} \\
J_{+} & u-J_{3}
\end{array}\right) .
$$

The auxiliary spinors enabled us to reproduce this well-known result in a remarkably simple and explicit way. They saved us from the need to construct projectors which single out irreducible representations and which are inevitable in the standard formulation.

Now we are going to describe another way for deriving the L-operator (2.55) by means of the fusion procedure. The main reason to embark upon one more calculation is that it can be generalized easily to the case of $q$-deformation (see Section 3.4) and, more importantly, to the elliptic deformation [10]. As before we deal with the symbols of finite-dimensional operators. The new ingredient is a factorization of the L-operator (cf. (2.15)). For calculating the symbol $\mathrm{R}(u \mid \lambda, \mu)$ of the "fused" R-matrices (2.50)

$$
\mathrm{R}(u \mid \lambda, \mu)=\langle\lambda|\mathrm{R}(u)| \mu\rangle\langle\lambda|\mathrm{R}(u-1)| \mu\rangle \cdots\langle\lambda|\mathrm{R}(u-n+1)| \mu\rangle,
$$

we choose the parametrization of the auxiliary spinor $\lambda_{1}=-z, \lambda_{2}=1$ from the very beginning. Remind a realization of the spin $\frac{1}{2}$ generators as differential operators (cf. (2.48))

$$
J_{+}=z^{2} \partial-z, \quad J_{-}=-\partial, \quad J_{3}=z \partial-\frac{1}{2},
$$

which act in the two-dimensional space of linear functions $\psi(z)=a_{1} z+a_{0}$. In the basis $\mathbf{e}_{1}=-z$, $\mathbf{e}_{2}=1$ of this space the matrices of the generators coincide with the Pauli-matrices

$$
\begin{aligned}
& J_{ \pm}\left(\mathbf{e}_{1}, \mathbf{e}_{2}\right)=\left(J_{ \pm} \mathbf{e}_{1}, J_{ \pm} \mathbf{e}_{2}\right)=\left(\mathbf{e}_{1}, \mathbf{e}_{2}\right) \sigma_{ \pm}, \\
& J_{3}\left(\mathbf{e}_{1}, \mathbf{e}_{2}\right)=\left(J_{3} \mathbf{e}_{1}, J_{3} \mathbf{e}_{2}\right)=\left(\mathbf{e}_{1}, \mathbf{e}_{2}\right) \frac{1}{2} \sigma_{3} .
\end{aligned}
$$

Next we use the fusion procedure and derive the Lax operator (2.55) together with a representation of the spin $\frac{n}{2}$ generators $(2.48)$ acting in the $(n+1)$-dimensional space of polynomials $\psi(z)=a_{n} z^{n}+\cdots+a_{0}$.

The symbol $\langle\lambda|\mathrm{R}(u)| \mu\rangle$ of Yang's R-matrix has been already found above (2.52), but now we are going to rewrite it in a different form. We represent it as a differential operator in the spinor variables acting on the identity operator symbol. Indeed, let us rewrite relations (2.57) in the equivalent form

$$
(-z, 1) \sigma_{ \pm}=J_{ \pm}(-z, 1), \quad(-z, 1) \frac{1}{2} \sigma_{3}=J_{3}(-z, 1),
$$

and use these formulae for calculating the symbol of Yang's R-matrix (2.49)

$$
\begin{aligned}
\langle\lambda|\mathrm{R}(u)| \mu\rangle & =\left(\begin{array}{cc}
(-z, 1)\left(u+\frac{1}{2}+\frac{1}{2} \sigma_{3}\right)|\mu\rangle & (-z, 1) \sigma_{-}|\mu\rangle \\
(-z, 1) \sigma_{+}|\mu\rangle & (-z, 1)\left(u+\frac{1}{2}-\frac{1}{2} \sigma_{3}\right)|\mu\rangle
\end{array}\right) \\
& =\left(\begin{array}{cc}
u+z \partial & -\partial \\
z^{2} \partial-z & u+1-z \partial
\end{array}\right)\left(\mu_{2}-\mu_{1} z\right) .
\end{aligned}
$$


We obtained the spin $\ell=\frac{1}{2}$ L-operator (2.35) (with the shifted spectral parameter $u \rightarrow u+\frac{1}{2}$ ) acting on the symbol of the identity operator $\langle\lambda \mid \mu\rangle=\left(\mu_{2}-\mu_{1} z\right)$. Then we observe that this symbol can be cast in the factorized form

$$
\langle\lambda|\mathrm{R}(u)| \mu\rangle=\left.\left(\begin{array}{cc}
1 & 0 \\
z & u+1
\end{array}\right)\left(\begin{array}{cc}
1 & -\partial_{1} \\
0 & 1
\end{array}\right)\left(\begin{array}{cc}
u & 0 \\
-z & 1
\end{array}\right)\left(\mu_{2}-\mu_{1} z_{1}\right)\right|_{z_{1}=z},
$$

which is easily checked by a direct calculation. Note that the factorization in (2.59) is slightly different from (2.15) (at $\ell=\frac{1}{2}$ ), since it involves a particular ordering of $z$ and $\partial$ and such ordering is compatible with the factorization of the L-operator up to the shift of spectral parameter.

Then we consider the product of two consecutive symbols in (2.56) and profit a lot from the factorization (2.59) which provides cancellation of two adjacent matrix factors (which are underlined in the following formula)

$$
\begin{aligned}
\langle\lambda|\mathrm{R}(u)| \mu\rangle\langle\lambda|\mathrm{R}(u-1)| \mu\rangle=\left(\begin{array}{cc}
1 & 0 \\
z & u+1
\end{array}\right)\left(\begin{array}{cc}
1 & -\partial_{1} \\
0 & 1
\end{array}\right) \underline{\left(\begin{array}{cc}
u & 0 \\
-z & 1
\end{array}\right)\left(\begin{array}{ll}
1 & 0 \\
z & u
\end{array}\right)} \\
\quad \times\left.\left(\begin{array}{cc}
1 & -\partial_{2} \\
0 & 1
\end{array}\right)\left(\begin{array}{cc}
u-1 & 0 \\
-z & 1
\end{array}\right)\left(\mu_{2}-\mu_{1} z_{1}\right)\left(\mu_{2}-\mu_{1} z_{2}\right)\right|_{z_{1}=z_{2}=z} \\
=\left.u\left(\begin{array}{cc}
1 & 0 \\
z & u+1
\end{array}\right)\left(\begin{array}{cc}
1 & -\partial_{1}-\partial_{2} \\
0 & 1
\end{array}\right)\left(\begin{array}{cc}
u-1 & 0 \\
-z & 1
\end{array}\right)\left(\mu_{2}-\mu_{1} z_{1}\right)\left(\mu_{2}-\mu_{1} z_{2}\right)\right|_{z_{1}=z_{2}=z} .
\end{aligned}
$$

By now the generalization of the previous result to the product of $n-1$ symbols (2.56) is evident

$$
\begin{aligned}
& \langle\lambda|\mathrm{R}(u)| \mu\rangle\langle\lambda|\mathrm{R}(u-1)| \mu\rangle \cdots\langle\lambda|\mathrm{R}(u-n+1)| \mu\rangle \\
& =r_{n}(u)\left(\begin{array}{cc}
1 & 0 \\
z & u+1
\end{array}\right)\left(\begin{array}{cc}
1 & -\partial_{1}-\partial_{2}-\cdots-\partial_{n} \\
0 & 1
\end{array}\right)\left(\begin{array}{cc}
u-n+1 & 0 \\
-z & 1
\end{array}\right) \\
& \quad \times\left.\left(\mu_{2}-\mu_{1} z_{1}\right) \cdots\left(\mu_{2}-\mu_{1} z_{n}\right)\right|_{z_{1}=\cdots=z_{n}=z} .
\end{aligned}
$$

Further we multiply all matrices on the right-hand side of the previous formula and obtain

$$
\begin{aligned}
r_{n}(u) & \left(\begin{array}{cc}
u-n+1+z\left(\partial_{1}+\cdots+\partial_{n}\right) & -\partial_{1}-\cdots-\partial_{n} \\
z^{2}\left(\partial_{1}+\cdots+\partial_{n}\right)-n z & u+1-z\left(\partial_{1}+\cdots+\partial_{n}\right)
\end{array}\right) \\
& \times\left.\left(\mu_{2}-\mu_{1} z_{1}\right) \cdots\left(\mu_{2}-\mu_{1} z_{n}\right)\right|_{z_{1}=\cdots=z_{n}=z} \\
= & r_{n}(u)\left(\begin{array}{cc}
u-n+1+z \partial & -\partial \\
z^{2} \partial-n z & u+1-z \partial
\end{array}\right)\left(\mu_{2}-\mu_{1} z\right)^{n},
\end{aligned}
$$

where on the last step we use an obvious formula

$$
\left.\left(\partial_{1}+\cdots+\partial_{n}\right)\left(\mu_{2}-\mu_{1} z_{1}\right) \cdots\left(\mu_{2}-\mu_{1} z_{n}\right)\right|_{z_{1}=\cdots=z_{n}=z}=\partial\left(\mu_{2}-\mu_{1} z\right)^{n} \text {. }
$$

The final result for the symbol (2.56) of the "fused" Yang R-matrices is

$$
\mathrm{R}(u \mid \lambda, \mu)=r_{n}(u)\left(\begin{array}{cc}
u+1-\frac{n}{2}+J_{3} & J_{-} \\
J_{+} & u+1-\frac{n}{2}-J_{3}
\end{array}\right)\left(\mu_{2}-\mu_{1} z\right)^{n},
$$

where the generators $J_{ \pm}, J_{3}$ for the representation of spin $\frac{n}{2}$ are given by (2.48).

Factorization of the L-operator plays an important role in the construction of the general R-operator for deformed [13, 16] and non-deformed [13] rank 1 symmetry algebra, as well as in the higher rank case [15]. Here we see that it finds a natural place in the fusion construction as well. 


\subsection{Fusion construction for $\mathrm{SL}(2, \mathbb{C})$}

The fusion procedure enables one to produce even more intricate $\mathfrak{s l}_{2}$-symmetric solutions of the YBE. Starting from the L-operator acting in the tensor product of spin $\frac{1}{2}$ and spin $\ell$ representations (we assume $\ell$ to be generic such that the corresponding representation is infinitedimensional) one obtains the R-operator which acts in the tensor product of spin $\frac{n}{2}$ and spin $\ell$ representations. Since we are mainly interested in the R-operators which are invariant with respect to the $\mathrm{SL}(2, \mathbb{C})$ group, we will thoroughly study how the fusion procedure applies in this case.

As before we profit a lot from the auxiliary spinors notation. However, from now on the holomorphic and antiholomorphic sectors are present and we need to introduce a pair of auxiliary spinors $\lambda_{i}, \bar{\lambda}_{\bar{i}}$. They are independent variables not related by the complex conjugation. Thus we introduce a pair of scalar objects (without spinor indices)

$$
\Lambda(u, \lambda, \mu)=\lambda_{i} \mathrm{~L}_{i}^{j}(u) \mu_{j}, \quad \bar{\Lambda}(\bar{u}, \bar{\lambda}, \bar{\mu})=\bar{\lambda}_{\bar{i}} \overline{\mathrm{L}}_{\bar{i}}^{\bar{j}}(\bar{u}) \bar{\mu}_{\bar{j}},
$$

which are linear combinations of the L-operators' entries (2.15), (2.16). An easy calculation shows that ${ }^{1}$

$$
\begin{aligned}
\Lambda(u, \lambda, \mu) & =-\left(\lambda_{1}+\lambda_{2} z\right)\left(\mu_{2}-\mu_{1} z\right) \partial+u_{2} \lambda_{2}\left(\mu_{2}-\mu_{1} z\right)-\left(u_{1}+1\right) \mu_{1}\left(\lambda_{1}+\lambda_{2} z\right) \\
& =-\left(\lambda_{1}+\lambda_{2} z\right)^{u_{2}+1}\left(\mu_{2}-\mu_{1} z\right)^{-u_{1}} \cdot \partial \cdot\left(\lambda_{1}+\lambda_{2} z\right)^{-u_{2}}\left(\mu_{2}-\mu_{1} z\right)^{u_{1}+1} .
\end{aligned}
$$

The factorized expression (2.61) looks much like formula (2.15). Indeed in both expressions the differential operators are sandwiched between some multiplication by a function operators. The analogous relation takes place for $\overline{\mathrm{L}}(2.16)$. Then we multiply a number of $\Lambda$-operators with shifted spectral parameters to form a $\Lambda$-string

$$
\begin{aligned}
\Lambda(u) \Lambda & (u-1) \cdots \Lambda(u-n+1)=(-1)^{n}\left(\lambda_{1}+\lambda_{2} z\right)^{u_{2}+1}\left(\mu_{2}-\mu_{1} z\right)^{-u_{1}} \\
& \times\left(\partial\left(\mu_{2}-\mu_{1} z\right)^{2}\right)^{n} \cdot\left(\lambda_{1}+\lambda_{2} z\right)^{-u_{2}+n-1}\left(\mu_{2}-\mu_{1} z\right)^{u_{1}-n}= \\
= & (-1)^{n}\left(\lambda_{1}+\lambda_{2} z\right)^{u_{2}+1}\left(\mu_{2}-\mu_{1} z\right)^{-u_{1}+n-1} \cdot \partial^{n} \cdot\left(\lambda_{1}+\lambda_{2} z\right)^{-u_{2}+n-1}\left(\mu_{2}-\mu_{1} z\right)^{u_{1}+1} .
\end{aligned}
$$

Here we apply the formula which can be easily proven by induction,

$$
\left(\partial\left(\mu_{2}-\mu_{1} z\right)^{2}\right)^{n}=\left(\mu_{2}-\mu_{1} z\right)^{n-1} \partial^{n}\left(\mu_{2}-\mu_{1} z\right)^{n+1} .
$$

Then we take into account the anti-holomorphic sector and form the product of $\Lambda$ - and $\bar{\Lambda}$-strings resulting in the symbol for a higher-spin R-operator

$$
\begin{aligned}
& \mathrm{R}_{\text {fus }}(u, \bar{u} \mid \lambda, \bar{\lambda}, \mu, \bar{\mu})=\Lambda(u) \Lambda(u-1) \cdots \Lambda(u-n+1) \bar{\Lambda}(\bar{u}) \bar{\Lambda}(\bar{u}-1) \cdots \bar{\Lambda}(\bar{u}-\bar{n}+1) \\
& =(-1)^{n+\bar{n}}\left[\lambda_{1}+\lambda_{2} z\right]^{u_{2}+1}\left[\mu_{2}-\mu_{1} z\right]^{-u_{1}+n-1} \cdot\left[\partial_{z}\right]^{n} \cdot\left[\lambda_{1}+\lambda_{2} z\right]^{-u_{2}+n-1}\left[\mu_{2}-\mu_{1} z\right]^{u_{1}+1} .
\end{aligned}
$$

This R-operator acts in the tensor product of the infinite-dimensional representation specified by the spins $\ell, \bar{\ell}$ and the finite-dimensional representation with the spins $\frac{n}{2}, \frac{\bar{n}}{2}$. Let us remind that $R_{\text {fus }}$ is a symbol with respect to the first finite-dimensional space only, but it is a differential operator in the second infinite-dimensional space. Evidently, the right-hand side of (2.62) is polynomial in $\lambda$ and $\mu$, as it should be. In order to reconstruct the operator itself from its symbol we resort to the rule (2.42). More precisely, we apply the corresponding relation to a function $\Phi(\lambda, \bar{\lambda} \mid z, \bar{z})$, which is homogeneous in $\lambda$ and $\bar{\lambda}$ of the homogeneity degree $n$ and $\bar{n}$, respectively,

$$
\left[\mathrm{R}_{\text {fus }}(u, \bar{u}) \Phi\right](\lambda, \bar{\lambda} \mid z, \bar{z})=\left.\mathrm{R}_{\text {fus }}\left(u, \bar{u} \mid \lambda, \bar{\lambda}, \partial_{\mu}, \partial_{\bar{\mu}}\right) \Phi(\mu, \bar{\mu} \mid z, \bar{z})\right|_{\mu=\bar{\mu}=0} .
$$

\footnotetext{
${ }^{1}$ We should note that the idea to reformulate the fusion procedure with the help of such $\Lambda$-operators belongs to D. Karakhanyan.
} 
We stress that the fusion formulae (2.62), (2.63) are somewhat different from the standard ones. We find them better adapted for applications.

The higher-spin R-operator (2.63) obtained by means of the fusion is identical with the reduction of the general R-operator calculated in (2.32), which will be demonstrated shortly. First of all the operator form of the star-triangle relation (2.28) enables one to rewrite the symbol (2.62) as follows

$$
\begin{aligned}
\mathrm{R}_{\text {fus }}(u, \bar{u} \mid \lambda, \bar{\lambda}, \mu, \bar{\mu})= & (-1)^{n+\bar{n}}\left[\lambda_{1}+\lambda_{2} z\right]^{u_{2}+1} \\
& \times\left[\partial_{z}\right]^{u_{1}+1} \cdot\left[\mu_{2}-\mu_{1} z\right]^{n} \cdot\left[\partial_{z}\right]^{-u_{1}+n-1}\left[\lambda_{1}+\lambda_{2} z\right]^{-u_{2}+n-1} .
\end{aligned}
$$

We have seen above that $\left(\lambda_{1}+\lambda_{2} x\right)^{n}$ is a generating function of the $(n+1)$-dimensional JordanSchwinger representation of $\mathfrak{s l}_{2}$. Its generalization to the group $\operatorname{SL}(2, \mathbb{C})$ is straightforward: $\left[\lambda_{1}+\lambda_{2} x\right]^{n}$ (see (2.9)) is a generating function of the $(n+1)(\bar{n}+1)$-dimensional representation realized in the space of homogeneous functions $\Psi(\lambda, \bar{\lambda})$,

$$
\Psi(\lambda, \bar{\lambda})=\Psi\left(\lambda_{1}, \lambda_{2}, \bar{\lambda}_{1}, \bar{\lambda}_{2}\right), \quad \Psi\left(\alpha \lambda_{1}, \alpha \lambda_{2}, \bar{\alpha} \bar{\lambda}_{1}, \bar{\alpha} \bar{\lambda}_{2}\right)=\alpha^{n} \bar{\alpha}^{\bar{n}} \Psi(\lambda, \bar{\lambda}) .
$$

Then we act by $\mathrm{R}_{\text {fus }}$ on the generating function according to (2.63) and choose the symbol in the form (2.64). At the same time we do not act by the $\mathrm{R}_{\text {fus }}$-operator on any function in its second space. At this point we take into account that

$$
\left[\partial_{\mu_{2}}-\partial_{\mu_{1}} z\right]^{n}\left[\mu_{1}+\mu_{2} x\right]^{n}=n ! \bar{n} ![x-z]^{n}
$$

and obtain

$$
\begin{aligned}
\mathrm{R}_{\mathrm{fus}} & \left(u+\frac{n}{2}, \bar{u}+\frac{\bar{n}}{2}\right)\left[\lambda_{1}+\lambda_{2} x\right]^{n} \\
& =n ! \bar{n} !\left[\lambda_{1}+\lambda_{2} z\right]^{u+\frac{n}{2}+\ell+1}\left[\partial_{z}\right]^{u+\frac{n}{2}-\ell}[z-x]^{n}\left[\partial_{z}\right]^{-u+\frac{n}{2}+\ell}\left[\lambda_{1}+\lambda_{2} z\right]^{-u+\frac{n}{2}-\ell-1} \\
& =n ! \bar{n} !\left[\lambda_{1}+\lambda_{2} z\right]^{u+\frac{n}{2}+\ell+1}[z-x]^{-u+\frac{n}{2}+\ell}\left[\partial_{z}\right]^{n}[z-x]^{u+\frac{n}{2}-\ell}\left[\lambda_{1}+\lambda_{2} z\right]^{-u+\frac{n}{2}-\ell-1} .
\end{aligned}
$$

Here we profited from the star-triangle relation (2.28) at the last step. In order to compare the reduction of the general R-operator (2.32) with the expression (2.66) following from the fusion formula (2.62) we just need to pass from the Jordan-Schwinger representation to the standard representation of $\mathrm{SL}(2, \mathbb{C})$ (in the space of functions of one complex variable) described in Section 2.1. Consequently we choose $\lambda_{1}=-z_{1}, \bar{\lambda}_{1}=-\bar{z}_{1}, \lambda_{2}=\bar{\lambda}_{2}=1$ and denote $z=z_{2}$, $\bar{z}=\bar{z}_{2}$. Finally, we see that both formulae are identical up to a numerical normalization.

We conclude that both ways to construct the higher-spin finite-dimensional (in one of the spaces) R-operators give identical results, and the general R-operator (2.22) contains all solutions of the Yang-Baxter equation associated with the principal series representations of the $\operatorname{SL}(2, \mathbb{C})$ group.

\section{The Faddeev modular double}

Using the patterns of the previous sections, in the following we show that all described constructions for the group $\mathrm{SL}(2, \mathbb{C})$ can be straightforwardly adapted to the modular double. Additionally, we construct corresponding finite-dimensional solutions of the YBE using the fusion.

\subsection{Representations of the quantum algebra}

The modular double of $\mathcal{U}_{q}\left(\mathfrak{s l}_{2}\right)$ was introduced by Faddeev in [18]. This algebra is formed by two sets of generators $\mathbf{E}, \mathbf{F}, \mathbf{K}$ and $\widetilde{\mathbf{E}}, \widetilde{\mathbf{F}}, \widetilde{\mathbf{K}}$. The usual commutation relations for $\mathbf{E}, \mathbf{F}, \mathbf{K}$ which generate $\mathcal{U}_{q}\left(\mathfrak{s l}_{2}\right)$ with $q=e^{i \pi \tau}(\tau \in \mathbb{C}$ and it is not a rational number)

$$
[\mathbf{E}, \mathbf{F}]=\frac{\mathbf{K}^{2}-\mathbf{K}^{-2}}{q-q^{-1}}, \quad \mathbf{K E}=q \mathbf{E K}, \quad \mathbf{K F}=q^{-1} \mathbf{F K}
$$


are supplemented by similar relations for $\widetilde{\mathbf{E}}, \widetilde{\mathbf{F}}, \widetilde{\mathbf{K}}$ with the deformation parameter $\widetilde{q}=e^{i \pi / \tau}$. The generators $\mathbf{E}$ and $\mathbf{F}$ commute with $\widetilde{\mathbf{E}}$ and $\widetilde{\mathbf{F}}$. The generator $\mathbf{K}$ anti-commutes with $\widetilde{\mathbf{E}}$ and $\widetilde{\mathbf{F}}, \widetilde{\mathbf{K}}$ anti-commutes with $\mathbf{E}$ and $\mathbf{F}$ while $\mathbf{K}$ commutes with $\widetilde{\mathbf{K}}$.

For particular representations of the modular double see $[4,18,20,35,40]$ and references therein. We use the parametrization $\tau=\frac{\omega^{\prime}}{\omega}$, where $\omega$ and $\omega^{\prime}$ are complex numbers with the positive imaginary parts, $\operatorname{Im} \omega>0, \operatorname{Im} \omega^{\prime}>0$, satisfying the normalization condition $\omega \omega^{\prime}=-\frac{1}{4}$. Then

$$
q=\exp \left(i \pi \omega^{\prime} / \omega\right), \quad \widetilde{q}=\exp \left(i \pi \omega / \omega^{\prime}\right)
$$

and the change $q \rightleftarrows \widetilde{q}$ is equivalent to $\omega \rightleftarrows \omega^{\prime}$. We denote also

$$
\omega^{\prime \prime}=\omega+\omega^{\prime}, \quad \beta=\frac{\pi}{12}\left(\frac{\omega}{\omega^{\prime}}+\frac{\omega^{\prime}}{\omega}\right) .
$$

In the following we deal with a representation $\pi_{s}$ of the modular double when the generators $\mathbf{K}_{s}=\pi_{s}(\mathbf{K}), \mathbf{E}_{s}=\pi_{s}(\mathbf{E}), \mathbf{F}_{s}=\pi_{s}(\mathbf{F})$ are realized as finite-difference operators acting on the space of entire functions rapidly decaying at infinity along contours parallel to the real line. This representation is parameterized by one complex parameter $s$ called the spin, and the generators have the following explicit form $[4,5,6]$

$$
\begin{aligned}
& \mathbf{K}_{s}=e^{-\frac{i \pi}{2 \omega} \hat{p}}, \quad\left(q-q^{-1}\right) \mathbf{E}_{s}=e^{\frac{i \pi x}{\omega}}\left[e^{-\frac{i \pi}{2 \omega}\left(\hat{p}-s-\omega^{\prime \prime}\right)}-e^{\frac{i \pi}{2 \omega}\left(\hat{p}-s-\omega^{\prime \prime}\right)}\right], \\
& \left(q-q^{-1}\right) \mathbf{F}_{s}=e^{-\frac{i \pi x}{\omega}}\left[e^{\frac{i \pi}{2 \omega}\left(\hat{p}+s+\omega^{\prime \prime}\right)}-e^{-\frac{i \pi}{2 \omega}\left(\hat{p}+s+\omega^{\prime \prime}\right)}\right]
\end{aligned}
$$

where $\hat{p}$ denotes a momentum operator in the coordinate representation $\hat{p}=\frac{1}{2 \pi i} \partial_{x}$. The formulae for generators $\widetilde{\mathbf{K}}_{s}, \widetilde{\mathbf{E}}_{s}, \widetilde{\mathbf{F}}_{s}$ are obtained by a simple interchange $\omega \rightleftarrows \omega^{\prime}$ in (3.3).

The modular double is associated with two basic special functions. The first one is the non-compact quantum dilogarithm which has the following integral representation

$$
\gamma(z)=\exp \left(-\frac{1}{4} \int_{-\infty}^{+\infty} \frac{d t}{t} \frac{e^{i t z}}{\sin (\omega t) \sin \left(\omega^{\prime} t\right)}\right),
$$

where the contour goes above the singularity at $t=0$. In the context of quantum integrable systems it has been found first in [19]. Some basic formulae for $\gamma(z)$ can be found in [20, 42]. This function respects a pair of finite-difference equations of the first order and the reflection relation

$$
\frac{\gamma\left(z+\omega^{\prime}\right)}{\gamma\left(z-\omega^{\prime}\right)}=1+e^{-\frac{i \pi}{\omega} z}, \quad \frac{\gamma(z+\omega)}{\gamma(z-\omega)}=1+e^{-\frac{i \pi}{\omega^{\prime}} z}, \quad \gamma(z) \gamma(-z)=e^{i \beta} e^{i \pi z^{2}}
$$

One can interpret $2 \omega$ and $2 \omega^{\prime}$ as some quasiperiods of the quantum dilogarithm.

The second function we need is

$$
D_{a}(z)=e^{-2 \pi i a z} \frac{\gamma(z+a)}{\gamma(z-a)} .
$$

In fact it coincides with the Faddeev-Volkov R-matrix [2, 43]. Some relations for this functions are presented in [5]. It naturally arises when one looks for the intertwining operator of equivalent representations of the modular double [35], and it serves as the main building block in the construction of a general R-matrix as an integral operator [5,6]. This general R-operator is 
a product of four Faddeev-Volkov's R-matrices. The function $D_{a}(z)$ obeys simple reflection relations

$$
D_{a}(z)=D_{a}(-z), \quad D_{a}(z) D_{-a}(z)=1,
$$

and a pair of finite-difference equations of the first order

$$
\frac{D_{a}\left(z-\omega^{\prime}\right)}{D_{a}\left(z+\omega^{\prime}\right)}=\frac{\cos \frac{\pi}{2 \omega}(z-a)}{\cos \frac{\pi}{2 \omega}(z+a)}, \quad \frac{D_{a}(z-\omega)}{D_{a}(z+\omega)}=\frac{\cos \frac{\pi}{2 \omega^{\prime}}(z-a)}{\cos \frac{\pi}{2 \omega^{\prime}}(z+a)} .
$$

Note that the functions $\gamma(z)$ and $D_{a}(z)$ are symmetric with respect to $\omega$ and $\omega^{\prime}$.

A generalization of the Faddeev-Volkov model still associated with the $\gamma(z)$-function was found in [38]. It leads to a more general R-operator than we consider here [11], which can be obtained as a limit from the most complicated known R-operator derived in [16].

Now we proceed to finite-dimensional representations of the modular double. In order to fix the spin $s_{0}$ such that a finite-dimensional representation decouples from the infinite-dimensional representation $\pi_{s_{0}}$ we resort to the intertwining operator of equivalent representations of the modular double. It is known that the representations $\pi_{s}$ and $\pi_{-s}$ are equivalent. The corresponding intertwining operator [35] is expressed in terms of the special function (3.6), such that

$$
D_{-s}(\hat{p}) \mathbf{K}_{s}=\mathbf{K}_{-s} D_{-s}(\hat{p}), \quad D_{-s}(\hat{p}) \mathbf{E}_{s}=\mathbf{E}_{-s} D_{-s}(\hat{p}), \quad D_{-s}(\hat{p}) \mathbf{F}_{s}=\mathbf{F}_{-s} D_{-s}(\hat{p}),
$$

where $\hat{p}$ is the momentum operator. There are analogous relations for $\widetilde{\mathbf{E}}, \widetilde{\mathbf{F}}, \widetilde{\mathbf{K}}$, since the $D$ function is invariant with respect to the permutation of $\omega$ and $\omega^{\prime}$. The latter relations can be easily checked using equations (3.8). Applying the Fourier transformation of the $D$-function $(3.6)[5,20,42]$

$$
\begin{aligned}
& A(a) \int_{-\infty}^{+\infty} d t e^{2 \pi i t z} D_{a}(t)=D_{-\omega^{\prime \prime}-a}(z), \\
& A(a) \equiv \frac{e^{\frac{i \pi}{2}\left(2 a+\omega^{\prime \prime}\right)^{2}+\frac{i \beta}{2}}}{\gamma\left(2 a+\omega^{\prime \prime}\right)}, \quad A(a) A\left(-a-\omega^{\prime \prime}\right)=1,
\end{aligned}
$$

we immediately represent the intertwiner $D_{-s}(\hat{p})$ as an integral operator (in analogy with (2.5))

$$
D_{-s}(\hat{p}) \Phi(x)=A\left(s-\omega^{\prime \prime}\right) \int_{-\infty}^{+\infty} d x^{\prime} D_{s-\omega^{\prime \prime}}\left(x-x^{\prime}\right) \Phi\left(x^{\prime}\right) .
$$

Thus the intertwiner admits two forms for generic values of $s$ : as a formal function of the momentum operator and a well-defined integral operator.

A finite $(n+1)(m+1)$-dimensional representation decouples from the infinite-dimensional one for special values of the spin

$$
s=s_{0}:=-\omega^{\prime \prime}-n \omega-m \omega^{\prime}, \quad n, m \in \mathbb{Z}_{\geq 0},
$$

where the integers $n$ and $m$ enumerate the points of a quarter-infinite lattice on the complex plane (or a line, for real $\left.\omega / \omega^{\prime}\right)$. Such finite-dimensional representations emerged first in the two-dimensional rational conformal field theory through $6 j$-symbols for the modular double with $q=e^{2 \pi i m /(m+1)}$ and $\tilde{q}=e^{2 \pi i(m+1) / m}, m \in \mathbb{Z}[22]$. The most general two-index $6 j$-symbols were discovered in the theory of elliptic hypergeometric functions [36]. They are described by meromorphic functions of one variable satisfying the biorthogonality relation with an absolutely 
continuous measure, which has the form usually ascribed to the functions of two independent variables. The latter property brought to the theory of spectral problems the new notion of two-index (bi-)orthogonality.

For the elliptic modular double the two-index finite-dimensional representations were discovered in [16, 17]. In principle, finite-dimensional representations of the Faddeev modular double can be derived as certain limits from this elliptic construction, but we give here an independent consideration and, moreover, describe the finite-dimensional R-matrices analogous to $(2.38)$. Note that in the $\mathrm{SL}(2, \mathbb{C})$ group case the finite-dimensional representations were also parametrized by a pair of non-negative integer numbers $n$ and $\bar{n}$, but the integer $\bar{n}$ has a different nature emerging from a discretization of the separate spin variable $\bar{s}$, which is absent in our case.

In order to find finite-dimensional representations of interest we investigate the null-space of the intertwiner. We take the formal operator identity

$$
D_{-s}(\hat{p}) D_{s}(\hat{p})=\mathbb{1}
$$

which is a consequence of the reflection formula (3.7), and rewrite it in an equivalent form substituting $D_{s}(\hat{p})$ and $\mathbb{1}$ for their kernels (see (3.11))

$$
D_{-s}(\hat{p}) D_{-s-\omega^{\prime \prime}}(x-y)=A^{-1}\left(-s-\omega^{\prime \prime}\right) \delta(x-y) .
$$

Then we note that zeros of the quantum dilogarithm $\gamma(z)=0$ are located at $z=\omega^{\prime \prime}+2 n \omega+2 m \omega^{\prime}$, $n, m \in \mathbb{Z}_{\geq 0}$, which indicates that the relation (3.12) is broken down at the corresponding points. Consequently for the spin values specified above, $s=s_{0}$, the right-hand side of (3.13) vanishes and a nontrivial null-space of $D_{-s_{0}}(\hat{p})$ arises

$$
D_{-s_{0}}(\hat{p}) D_{n \omega+m \omega^{\prime}}(x-y)=0, \quad n, m \in \mathbb{Z}_{\geq 0} .
$$

The latter formula is a deformed analogue of (2.14). From the intertwining relations (3.9) the null-space is seen to be invariant under the action of the modular double generators. Corresponding representation is finite-dimensional as we will see shortly. The generators are fixed by expressions (3.3) and their modular duals with the spin parameter $s=s_{0}$. One can also show that the corresponding representation is irreducible.

In this way we have found that $D_{n \omega+m \omega^{\prime}}(x-y)$ is the generating function of a finitedimensional representation containing all its basis vectors. Here $y$ is an auxiliary parameter, which is convenient to write in the exponential form

$$
Y=Y(y)=e^{\frac{i \pi}{2 \omega} y}, \quad \tilde{Y}=\tilde{Y}(y)=e^{\frac{i \pi}{2 \omega^{\prime}} y} .
$$

Since we assume that the quasiperiods are incommensurate (i.e., that $\tau=\frac{\omega^{\prime}}{\omega}$ is not a rational number), the auxiliary variables $Y$ and $\widetilde{Y}$ are multiplicatively incommensurate for generic $y$ (i.e., if $\tilde{Y}^{k} Y^{l}=1$ for some integer $k$ and $l$, then $k=l=0$ ). Using the finite-difference equations (3.8) our generating function can be rewritten as a finite product

$$
\begin{aligned}
D_{n \omega+m \omega^{\prime}}(x-y)= & \prod_{k=0}^{n-1}\left(\widetilde{Y}^{-1} \widetilde{X} \tilde{q}^{\frac{n-1}{2}-k}+\widetilde{Y} \widetilde{X}^{-1}(-1)^{m} \tilde{q}^{-\frac{n-1}{2}+k}\right) \\
& \times \prod_{l=0}^{m-1}\left(Y^{-1} X q^{\frac{m-1}{2}-l}+Y X^{-1}(-1)^{n} q^{-\frac{m-1}{2}+l}\right),
\end{aligned}
$$

where we use the shorthand notation

$$
X=X(x)=e^{\frac{i \pi}{2 \omega} x}, \quad \tilde{X}=\tilde{X}(x)=e^{\frac{i \pi}{2 \omega^{\prime}} x} .
$$


Expanding the Laurent polynomial (3.15) in integer powers of $Y(y)$ and $\tilde{Y}(y)$ we extract $(n+1)(m+1)$ basis elements of the finite-dimensional representation given by the monomials

$$
\widetilde{X}^{n-2 k} X^{m-2 l} \quad \text { with } \quad k=0,1, \ldots, n, \quad l=0,1, \ldots, m .
$$

Let us note that for $s=s_{0}$ the integral in (3.11) diverges. The divergence is compensated by the normalization factor, which turns to zero, $A\left(s_{0}-\omega^{\prime \prime}\right)=0$. The ambiguity can be resolved and for finite-dimensional representations the intertwiner $D_{-s_{0}}(\hat{p})$ becomes a sum of finite-difference operators which follows from (3.8). One can directly check as well that the basis vectors (3.17) are annihilated by $D_{-s_{0}}(\hat{p})$.

\subsection{An infinite-dimensional R-operator for the modular double}

Now we proceed to integrable structures for the modular double. The L-operator is constructed out of the modular double generators taken in the representation $\pi_{s}(3.3)$ [5],

$$
\mathrm{L}(u \mid s)=\left(\begin{array}{cc}
e^{\frac{i \pi}{\omega} u} \mathbf{K}_{s}-e^{-\frac{i \pi}{\omega} u} \mathbf{K}_{s}^{-1} & \left(q-q^{-1}\right) \mathbf{F}_{s} \\
\left(q-q^{-1}\right) \mathbf{E}_{s} & e^{\frac{i \pi}{\omega} u} \mathbf{K}_{s}^{-1}-e^{-\frac{i \pi}{\omega} u} \mathbf{K}_{s}
\end{array}\right) .
$$

This L-operator respects the standard RLL-intertwining relation (cf. (2.18))

$$
\mathrm{R}_{a b, e f}(u-v) \mathrm{L}_{e c}(u) \mathrm{L}_{f d}(v)=\mathrm{L}_{b f}(v) \mathrm{L}_{a e}(u) \mathrm{R}_{e f, c d}(u-v)
$$

with $4 \times 4$ trigonometric R-matrix (cf. (3.38))

$$
\mathrm{R}(u)=\frac{\sin \frac{i \pi}{\omega}\left(u+\frac{\omega^{\prime}}{2}\right)}{2 \sin \frac{i \pi \omega^{\prime}}{2 \omega}} \mathbb{1} \otimes \mathbb{1}+\frac{1}{2} \sigma_{1} \otimes \sigma_{1}+\frac{1}{2} \sigma_{2} \otimes \sigma_{2}+\frac{\cos \frac{i \pi}{\omega}\left(u+\frac{\omega^{\prime}}{2}\right)}{2 \cos \frac{i \pi \omega^{\prime}}{2 \omega}} \sigma_{3} \otimes \sigma_{3},
$$

which is equivalent to the set of commutation relations (3.1). The second L-operator is obtained from $\mathrm{L}(u)$ by the interchange $\omega \rightleftarrows \omega^{\prime}: \widetilde{\mathrm{L}}(u)=\left.\mathrm{L}(u)\right|_{\omega \rightleftarrows \omega^{\prime}}$. The same is true for the corresponding $\widetilde{\mathrm{R}}$-matrix. In the following we indicate formulae only for the L-operator (3.18), and all relations for the $\widetilde{\mathrm{L}}$-operator have the same form with $\omega \rightleftarrows \omega^{\prime}$.

The L-operator (3.18) can be represented in the factorized form

$$
\begin{aligned}
& \mathrm{L}(u \mid s)=\left(\begin{array}{cc}
U_{2} & -U_{2}^{-1} \\
-U_{2}^{-1} e^{\frac{i \pi}{\omega} x} & U_{2} e^{\frac{i \pi}{\omega} x}
\end{array}\right)\left(\begin{array}{cc}
e^{-\frac{i \pi}{2 \omega}\left(p-\omega^{\prime \prime}\right)} & 0 \\
0 & e^{\frac{i \pi}{2 \omega}\left(p-\omega^{\prime \prime}\right)}
\end{array}\right)\left(\begin{array}{cc}
-U_{1} & U_{1}^{-1} e^{-\frac{i \pi}{\omega} x} \\
-U_{1}^{-1} & U_{1} e^{-\frac{i \pi}{\omega} x}
\end{array}\right), \\
& U_{1}=e^{\frac{i \pi}{2 \omega} u_{1}}, \quad U_{2}=e^{\frac{i \pi}{2 \omega} u_{2}}
\end{aligned}
$$

where we introduced the "light-cone" parameters $u_{1}$ and $u_{2}$ instead of $u$ and $s$

$$
u_{1}=u+\frac{s}{2}+\frac{\omega}{2}-\frac{\omega^{\prime}}{2}, \quad u_{2}=u-\frac{s}{2}+\frac{\omega}{2}-\frac{\omega^{\prime}}{2} .
$$

In the notation $\mathrm{L}(u)$ we omit for simplicity dependence on the spin parameter $s$. Factorization of the L-operator of the XXZ spin chain has been introduced in [3] in relation to the chiral Potts models. The factorization formula (3.20) is completely analogous to formula (2.15) for the $\operatorname{SL}(2, \mathbb{C})$ group. The same can be said about spectral parameters $u_{1}, u_{2}$ in $(2.17)$ and (3.21). However, although the operators $\mathrm{L}(u), \widetilde{\mathrm{L}}(u)$ for the modular double look as analogues of $\mathrm{L}(u), \overline{\mathrm{L}}(\bar{u})$ for $\mathrm{SL}(2, \mathbb{C})$, in fact, they are different in their nature.

At the level of R-operators an analogy with the rational case persists as well. The general R-operator acts in the tensor product of two infinite-dimensional representations $\pi_{s_{1}} \otimes \pi_{s_{2}}(3.3)$. It has been found first in [5], but the corresponding form of the R-operator is not suitable for 
our purposes. Here we profit from another construction implemented in [6], where it has been obtained solving a pair of RLL-relations (cf. (2.19), (2.20)),

$$
\begin{aligned}
& \mathrm{R}_{12}(u-v) \mathrm{L}_{1}\left(u \mid s_{1}\right) \mathrm{L}_{2}\left(v \mid s_{2}\right)=\mathrm{L}_{1}\left(v \mid s_{2}\right) \mathrm{L}_{2}\left(u \mid s_{1}\right) \mathrm{R}_{12}(u-v), \\
& \mathrm{R}_{12}(u-v) \widetilde{\mathrm{L}}_{1}\left(u \mid s_{1}\right) \widetilde{\mathrm{L}}_{2}\left(v \mid s_{2}\right)=\widetilde{\mathrm{L}}_{1}\left(v \mid s_{2}\right) \widetilde{\mathrm{L}}_{2}\left(u \mid s_{1}\right) \mathrm{R}_{12}(u-v) .
\end{aligned}
$$

The spin parameters $s_{1}, s_{2}$ and the spectral parameters $u, v$ appearing in the RLL-relations (3.22), (3.23) are combined to four "light-cone" parameters $u_{1}, u_{2}, v_{1}, v_{2}$ in accordance with (3.21), i.e.,

$$
\begin{array}{ll}
u_{1}=u+\frac{s_{1}}{2}+\frac{\omega}{2}-\frac{\omega^{\prime}}{2}, & u_{2}=u-\frac{s_{1}}{2}+\frac{\omega}{2}-\frac{\omega^{\prime}}{2}, \\
v_{1}=v+\frac{s_{2}}{2}+\frac{\omega}{2}-\frac{\omega^{\prime}}{2}, & v_{2}=v-\frac{s_{2}}{2}+\frac{\omega}{2}-\frac{\omega^{\prime}}{2} .
\end{array}
$$

The notation $\mathrm{R}_{12}(u-v)$ is a shortened version of $\mathrm{R}_{12}\left(u-v \mid s_{1}, s_{2}\right)$ or $\mathrm{R}_{12}\left(u_{1}, u_{2} \mid v_{1}, v_{2}\right)$ taking into account the spin parameters.

The R-operator is invariant with respect to the modular double, i.e., it commutes with the co-product of the generators. More precisely,

$$
\begin{aligned}
& {[\mathrm{R}(u), \Delta(\mathbf{K})]=[\mathrm{R}(u), \Delta(\mathbf{E})]=[\mathrm{R}(u), \Delta(\mathbf{F})]=0,} \\
& {[\mathrm{R}(u), \Delta(\widetilde{\mathbf{K}})]=[\mathrm{R}(u), \Delta(\widetilde{\mathbf{E}})]=[\mathrm{R}(u), \Delta(\widetilde{\mathbf{F}})]=0,}
\end{aligned}
$$

where we abbreviate the co-product taken in the tensor of representations with spins $s_{1}$ and $s_{2}$, $\left(\pi_{s_{1}} \otimes \pi_{s_{2}}\right) \circ \Delta$, to $\Delta$ bearing in mind the specified representations. The co-product is given by the formulae

$$
\Delta(\mathbf{K})=\mathbf{K}_{1} \mathbf{K}_{2}, \quad \Delta(\mathbf{E})=\mathbf{E}_{1} \mathbf{K}_{2}+\mathbf{K}_{1}^{-1} \mathbf{E}_{2}, \quad \Delta(\mathbf{F})=\mathbf{F}_{1} \mathbf{K}_{2}+\mathbf{K}_{1}^{-1} \mathbf{F}_{2} .
$$

Analogous relations take place for $\widetilde{\mathbf{E}}, \widetilde{\mathbf{F}}, \widetilde{\mathbf{K}}$. The invariance (3.24) follows straightforwardly from the RLL-relations (3.22), (3.23) subject to the shift of spectral parameters $u \rightarrow u+w, v \rightarrow v+w$ with arbitrary $w$.

Construction of the general R-operator for the modular double from [6] follows the same pattern as for the $\mathrm{SL}(2, \mathbb{C})$ group. It is based on the elementary intertwining operators that yield an integral operator representation of the symmetric group $\mathfrak{S}_{4}$. Now we repeat with a slight modification what has been said in Section 2.2 about the $\mathrm{SL}(2, \mathbb{C})$-invariant R-operator. The general R-operator is a product of four factors which are elementary intertwining operators

$$
\mathrm{R}_{12}(u-v)=D_{u_{2}-v_{1}}\left(x_{12}\right) D_{u_{1}-v_{1}}\left(\hat{p}_{2}\right) D_{u_{2}-v_{2}}\left(\hat{p}_{1}\right) D_{u_{1}-v_{2}}\left(x_{12}\right) .
$$

Here we denote $x_{i j}=x_{i}-x_{j}$. The latter formula has to be compared with (2.22) which has the same structure, only the building blocks are different. The change $\omega \rightleftarrows \omega^{\prime}$ does not alter the R-operator (3.25) which satisfies both RLL-relations (3.22) and (3.23). Similar to the expression (2.22), the representation (3.25) for our infinite-dimensional R-operator plays a major role in what follows. In the next section we find its reductions to finite-dimensional invariant subspaces.

According to (3.25) the general R-operator is a product of four Faddeev-Volkov's R-matrices [43]. Applying (3.11) one can rewrite it explicitly as an integral operator. Let us note that it is not the only possible form of the R-operator. Initially constructed in [5] the R-operator for the modular double was obtained in the form which is not convenient enough to address the current problem. In [5] the R-operator appeared in disguise of the $D$-function (3.6) and arcosh of the Casimir operator. Thus, dealing with such an operator, one has to decompose tensor products to a sum of irreducible representations and use the Clebsch-Gordan coefficients [35]. The R-operator in the form (3.25) has the virtue of not demanding any auxiliary information. 
In order to justify the chosen terminology for the R-operator factors in (3.19), we indicate here the relations

$$
\begin{aligned}
& D_{u_{2}-u_{1}}\left(\hat{p}_{1}\right) \mathrm{L}_{1}\left(u_{1}, u_{2}\right)=\mathrm{L}_{1}\left(u_{2}, u_{1}\right) D_{u_{2}-u_{1}}\left(\hat{p}_{1}\right), \\
& D_{v_{2}-v_{1}}\left(\hat{p}_{2}\right) \mathrm{L}_{2}\left(v_{1}, v_{2}\right)=\mathrm{L}_{2}\left(v_{2}, v_{1}\right) D_{v_{2}-v_{1}}\left(\hat{p}_{2}\right), \\
& D_{u_{1}-v_{2}}\left(x_{12}\right) \mathrm{L}_{1}\left(u_{1}, u_{2}\right) \mathrm{L}_{2}\left(v_{1}, v_{2}\right)=\mathrm{L}_{1}\left(v_{2}, u_{2}\right) \mathrm{L}_{2}\left(v_{1}, u_{1}\right) D_{u_{1}-v_{2}}\left(x_{12}\right),
\end{aligned}
$$

which have a clear meaning in terms of the permutation group $\mathfrak{S}_{4}$ and which enable us to check the RLL-relations (3.22) and (3.23). Similar to (2.24) and (2.25), here we assume that the $D$-operators are acting as $2 \times 2$ diagonal matrices proportional to the unit matrix.

The elementary intertwining operators possess a number of peculiar properties. They satisfy the Coxeter relations (cf. (2.23))

$$
D_{a}\left(\hat{p}_{k}\right) D_{a+b}\left(x_{12}\right) D_{b}\left(\hat{p}_{k}\right)=D_{b}\left(x_{12}\right) D_{a+b}\left(\hat{p}_{k}\right) D_{a}\left(x_{12}\right), \quad k=1,2 .
$$

Using these relations one can check [6] that the R-operator (3.25) satisfies the YBE

$$
\mathrm{R}_{23}(u-v) \mathrm{R}_{12}(u) \mathrm{R}_{23}(v)=\mathrm{R}_{12}(v) \mathrm{R}_{23}(u) \mathrm{R}_{12}(u-v) .
$$

Both sides in the latter relation are endomorphisms on the space $\pi_{s_{1}} \otimes \pi_{s_{2}} \otimes \pi_{s_{3}}$. For brevity we do not indicate dependence on the spin parameters.

The Coxeter relations (3.28) are equivalent to the star-triangle relation [43] which has three manifestations:

1) an integral identity $[5,40,42]$

$$
\begin{aligned}
& A(a) A(b) A(c) \int_{-\infty}^{+\infty} d z D_{a}\left(z-z_{1}\right) D_{b}\left(z-z_{2}\right) D_{c}\left(z-z_{3}\right) \\
& =D_{-\omega^{\prime \prime}-a}\left(z_{2}-z_{3}\right) D_{-\omega^{\prime \prime}-b}\left(z_{3}-z_{1}\right) D_{-\omega^{\prime \prime}-c}\left(z_{1}-z_{2}\right) \quad \text { with } \quad a+b+c=-2 \omega^{\prime \prime} ;
\end{aligned}
$$

2) a particular point in the image of the operator $D_{-a-\omega^{\prime \prime}}\left(\hat{p}_{1}\right)$ (with the same restriction on the parameters as before)

$$
D_{-a-\omega^{\prime \prime}}\left(\hat{p}_{1}\right)\left(D_{b}\left(z_{12}\right) D_{c}\left(z_{13}\right)\right)=\frac{D_{-\omega^{\prime \prime}-a}\left(z_{23}\right) D_{-\omega^{\prime \prime}-b}\left(z_{13}\right) D_{-\omega^{\prime \prime}-c}\left(z_{12}\right)}{A(b) A(c)} ;
$$

3) an operator identity

$$
D_{a}(\hat{p}) D_{a+b}(x) D_{b}(\hat{p})=D_{b}(x) D_{a+b}(\hat{p}) D_{a}(x) .
$$

\subsection{Finite-dimensional reduction of the $\mathrm{R}$-operator}

Now we have all ingredients at hand to perform a reduction of the described R-operator for modular double to a finite-dimensional representation in one of its tensor factors. The calculation follows precisely the same pattern as in the $\mathrm{SL}(2, \mathbb{C})$ case (see Section 2.3). Again the principal possibility of this reduction is based on the following relation for the R-operator (3.25)

$$
D_{u_{2}-u_{1}}\left(\hat{p}_{1}\right) \mathbb{R}_{12}\left(u_{1}, u_{2} \mid v_{1}, v_{2}\right)=\mathbb{R}_{12}\left(u_{2}, u_{1} \mid v_{1}, v_{2}\right) D_{u_{2}-u_{1}}\left(\hat{p}_{1}\right),
$$

which can be proved using the identity (3.28). Here, again, $\mathbb{R}_{12}=\mathrm{P}_{12} \mathrm{R}_{12}$, where $\mathrm{P}_{12}$ is a permutation operator. This relation shows that both, the null-space of the intertwining operator $D_{u_{2}-u_{1}}\left(\hat{p}_{1}\right)$ and the image of the intertwining operator $D_{u_{1}-u_{2}}\left(\hat{p}_{1}\right)$, are mapped onto themselves by our R-matrix $\mathbb{R}_{12}$. Therefore the invariant finite-dimensional subspaces of the nullspace are invariant with respect to the action of the R-operator as well. 
We consider the R-operator $\mathrm{R}_{12}\left(u \mid s_{0}, s\right)$ acting on the tensor product $\pi_{s_{0}} \otimes \pi_{s}$ and introduce the "light-cone" parameters (see (3.21))

$$
\begin{aligned}
& u_{1}=u+\frac{s_{0}}{2}+\frac{\omega}{2}-\frac{\omega^{\prime}}{2}, \quad u_{2}=u-\frac{s_{0}}{2}+\frac{\omega}{2}-\frac{\omega^{\prime}}{2}, \\
& v_{1}=\frac{s}{2}+\frac{\omega}{2}-\frac{\omega^{\prime}}{2}, \quad v_{2}=-\frac{s}{2}+\frac{\omega}{2}-\frac{\omega^{\prime}}{2} .
\end{aligned}
$$

We apply the R-operator to the function $D_{-\omega^{\prime \prime}+u_{2}-u_{1}}\left(x_{13}\right)$ in the first space. For $s_{0}=-\omega^{\prime \prime}-$ $n \omega-m \omega^{\prime}, n, m=0,1,2, \ldots$, the latter function becomes a generating function of the finitedimensional representation in the first space. However, for a moment $s_{0}$ is assumed to be generic. According to the structure of R-operator (3.25) we consider sequential action of its separate factors. As the first step, we apply $D_{u_{2}-v_{2}}\left(\hat{p}_{1}\right) D_{u_{1}-v_{2}}\left(x_{12}\right)$ to $D_{-\omega^{\prime \prime}+u_{2}-u_{1}}\left(x_{13}\right) \Phi\left(x_{2}\right)$ and, using formula (3.30), obtain

$$
\begin{aligned}
& D_{u_{2}-v_{2}}\left(\hat{p}_{1}\right) D_{u_{1}-v_{2}}\left(x_{12}\right) D_{-\omega^{\prime \prime}+u_{2}-u_{1}}\left(x_{13}\right) \Phi\left(x_{2}\right) \\
& \quad=\frac{A\left(u_{1}-u_{2}\right)}{A\left(u_{1}-v_{2}\right)} \cdot D_{u_{1}-u_{2}}\left(x_{12}\right) D_{-\omega^{\prime \prime}+v_{2}-u_{1}}\left(x_{13}\right) D_{u_{2}-v_{2}}\left(x_{23}\right) \Phi\left(x_{2}\right) .
\end{aligned}
$$

Further we apply the third factor $D_{u_{1}-v_{1}}\left(\hat{p}_{2}\right)$ of the R-operator to both sides of this relation. On the right-hand sides we use the relation

$$
\begin{aligned}
& D_{u_{1}-v_{1}}\left(\hat{p}_{2}\right) D_{u_{1}-u_{2}}\left(x_{12}\right) D_{u_{2}-v_{2}}\left(x_{23}\right) \Phi\left(x_{2}\right) \\
& \quad=A^{-1}\left(u_{1}-v_{1}\right) A^{-1}\left(u_{1}-u_{2}\right) \cdot D_{u_{2}-u_{1}-\omega^{\prime \prime}}\left(\hat{p}_{1}\right) D_{v_{1}-u_{1}-\omega^{\prime \prime}}\left(x_{12}\right) D_{u_{2}-v_{2}}\left(x_{13}\right) \Phi\left(x_{1}\right),
\end{aligned}
$$

which can be easily checked taking into account the integral form of the intertwiner (3.11). In a full analogy with the $\mathrm{SL}(2, \mathbb{C})$ calculation we traded a complicated integral operator $D_{u_{1}-v_{1}}\left(\hat{p}_{2}\right)$ for $D_{u_{2}-u_{1}-\omega^{\prime \prime}}\left(\hat{p}_{1}\right)$ which turns to $D_{n \omega+m \omega^{\prime}}\left(\hat{p}_{1}\right)$ in the finite-dimensional setting. The latter operator is just a sum of the finite-difference operators which follows from equations (3.8). The substitution $x-y \rightarrow \hat{p}_{1}$ in (3.15) yields an explicit expression for $D_{n \omega+m \omega^{\prime}}\left(\hat{p}_{1}\right)$,

$$
\begin{aligned}
D_{n \omega+m \omega^{\prime}}(\hat{p})= & \prod_{k=0}^{n-1}\left(e^{\frac{i \pi}{2 \omega^{\prime}} \hat{p}} \tilde{q}^{\frac{n-1}{2}-k}+e^{-\frac{i \pi}{2 \omega^{\prime}} \hat{p}}(-1)^{m} \tilde{q}^{-\frac{n-1}{2}+k}\right) \\
& \times \prod_{l=0}^{m-1}\left(e^{\frac{i \pi}{2 \omega} \hat{p}} q^{\frac{m-1}{2}-l}+e^{-\frac{i \pi}{2 \omega} \hat{p}}(-1)^{n} q^{-\frac{m-1}{2}+l}\right) .
\end{aligned}
$$

The fourth factor of the R-operator is inert being the multiplication by a function operator.

Thus the integral R-operator for the modular double (3.25) acting on the tensor product of two infinite-dimensional representations $\pi_{s_{0}} \otimes \pi_{s}$ can be reduced to a finite-dimensional representation in the first space for $s_{0}=-\omega^{\prime \prime}-n \omega-m \omega^{\prime}, n, m \in \mathbb{Z}_{\geq 0}$. It acts on the generating function of finite-dimensional representation (3.15) according to the following explicit formula

$$
\begin{aligned}
& \mathbb{R}_{12}\left(u \mid s_{0}, s\right) \cdot D_{n \omega+m \omega^{\prime}}\left(x_{13}\right) \Phi\left(x_{2}\right)=c \cdot D_{u-\frac{s_{0}}{2}-\frac{s}{2}}\left(x_{12}\right) \\
& \quad \times D_{-u-\frac{s_{0}}{2}-\frac{s}{2}-\omega^{\prime \prime}}\left(x_{23}\right) \cdot D_{n \omega+m \omega^{\prime}}\left(\hat{p}_{2}\right) \cdot D_{-u-\frac{s_{0}}{2}+\frac{s}{2}-\omega^{\prime \prime}}\left(x_{12}\right) D_{u-\frac{s_{0}}{2}+\frac{s}{2}}\left(x_{23}\right) \Phi\left(x_{2}\right),
\end{aligned}
$$

where the normalization factor is

$$
c^{-1}=A\left(u+\frac{s_{0}}{2}+\frac{s}{2}\right) A\left(u+\frac{s_{0}}{2}-\frac{s}{2}\right)
$$

and $x_{3}$ is an auxiliary parameter.

Both sides of the equality (3.36) can be expanded in integer powers of the variables $X_{3}\left(x_{3}\right)$, $\widetilde{X}_{3}\left(x_{3}\right)$ (see $(3.16)$ ). This yields simultaneously an expansion in integer powers of the variables 
$X_{1}\left(x_{1}\right), \widetilde{X}_{1}\left(x_{1}\right)$ (see $(3.17)$ ), which form a basis of the finite-dimensional representation of interest. The resulting formula (3.36) is very helpful in applications. We use it as follows. Firstly, finite-difference operators in the sum $D_{n \omega+m \omega^{\prime}}\left(\hat{p}_{2}\right)$ act from the left on the $D$-functions and shift their arguments. After these shifts we trade all $D$-functions (3.6) in (3.36) for quantum dilogarithms (3.4) and apply the finite-difference equations (3.5). In this way we completely get rid off the quantum dilogarithms. The final result contains only trigonometric functions, i.e., a linear combination of the products of $X_{1}\left(x_{1}\right), \widetilde{X}_{1}\left(x_{1}\right), X_{3}\left(x_{3}\right), \widetilde{X}_{3}\left(x_{3}\right)$, and $\Phi\left(x_{2}\right)$ with the shifted argument. Thus the restriction of the general R-operator can be represented as an $(n+1)(m+1)$-dimensional matrix whose entries are finite-difference operators with the trigonometric coefficients.

Formula (3.36) constitutes one of the main results of this paper. It gives a new rich class of solutions of the YBE which are endomorphisms on a tensor product of finite-dimensional and infinite-dimensional representations of the modular double specified in (3.3).

In $[32,33]$ an explicit hypergeometric formula for the R-matrix of $U_{q}\left(\mathfrak{s l}_{2}\right)$ acting on a tensor product of two highest-weight representations has been presented. It would be interesting to relate our formula (3.36) to R-matrices from these papers. In [27] group theoretical origins of similar factorization formulae were elucidated from the representation theory of $U_{q}\left(\hat{\mathfrak{s}}_{2}\right)$ algebra.

In order to demonstrate how formula (3.36) works in practice we recover the L-operator (3.18) out of the R-operator (3.25). With this task in mind, we choose the spin $s_{0}=-\omega^{\prime}-\omega^{\prime \prime}$, i.e., fix $n=0, m=1$. The generating function (3.15) of the 2 -dimensional representation in the first space is

$$
D_{\omega^{\prime}}\left(x_{13}\right)=e^{\frac{i \pi}{2 \omega} x_{13}}+e^{-\frac{i \pi}{2 \omega} x_{13}} .
$$

Consequently $\mathbf{e}_{1}=e^{\frac{i \pi}{2 \omega} x_{1}}, \mathbf{e}_{2}=e^{-\frac{i \pi}{2 \omega} x_{1}}$ form a basis of $\mathbb{C}^{2}$. The finite-difference operator in (3.36) is $D_{\omega^{\prime}}\left(\hat{p}_{2}\right)=e^{\frac{i \pi}{2 \omega} \hat{p}_{2}}+e^{-\frac{i \pi}{2 \omega} \hat{p}_{2}}$. Up to a normalization factor the right-hand side of (3.36) takes the form

$$
\begin{aligned}
\cosh & \frac{i \pi}{2 \omega}\left(x_{12}-u+\frac{s}{2}-\frac{\omega}{2}\right) \cdot \cosh \frac{i \pi}{2 \omega}\left(x_{23}-u-\frac{s}{2}-\frac{\omega}{2}-\omega^{\prime}\right) \cdot \Phi\left(x_{2}-\omega^{\prime}\right) \\
& +\cosh \frac{i \pi}{2 \omega}\left(x_{12}+u-\frac{s}{2}+\frac{\omega}{2}\right) \cdot \cosh \frac{i \pi}{2 \omega}\left(x_{23}+u+\frac{s}{2}+\frac{\omega}{2}+\omega^{\prime}\right) \cdot \Phi\left(x_{2}+\omega^{\prime}\right)
\end{aligned}
$$

Expanding this function in terms of $X_{1}^{ \pm 1}\left(x_{1}\right) X_{3}^{ \pm 1}\left(x_{3}\right)=e^{\frac{i \pi}{2 \omega}\left( \pm x_{1} \pm x_{3}\right)}$ we obtain

$$
\begin{aligned}
& \mathbb{R}_{12}\left(u-\omega-\frac{\omega^{\prime}}{2}\right) \mathbf{e}_{1}=c \cdot\left[\mathbf{e}_{1}\left(e^{\frac{i \pi}{\omega} u} \mathbf{K}_{s}-e^{-\frac{i \pi}{\omega} u} \mathbf{K}_{s}^{-1}\right)+\mathbf{e}_{2}\left(q-q^{-1}\right) \mathbf{E}_{s}\right], \\
& \mathbb{R}_{12}\left(u-\omega-\frac{\omega^{\prime}}{2}\right) \mathbf{e}_{2}=c \cdot\left[\mathbf{e}_{1}\left(q-q^{-1}\right) \mathbf{F}_{s}+\mathbf{e}_{2}\left(e^{\frac{i \pi}{\omega} u} \mathbf{K}_{s}^{-1}-e^{-\frac{i \pi}{\omega} u} \mathbf{K}_{s}\right)\right] .
\end{aligned}
$$

Thus we have reproduced the desired result (3.18). In a similar way one reproduces the $\widetilde{\mathrm{L}}$ operator at $s_{0}=-\omega-\omega^{\prime \prime}$. Implementing these reduced R-operators in the YBE (3.29) one recovers the RLL-relations (3.23). An explicit matrix factorization formula for $\mathbb{R}_{12}\left(u \mid s_{0}, s\right)$ generalizing the L-operator factorization (3.20) was derived in the followup paper [7].

A reduction of the $\mathrm{R}$-operator to finite-dimensional representations in both spaces can be constructed as well. One just should choose an appropriate discrete value of the spin $s$ in (3.36) and substitute $\Phi\left(x_{2}\right)$ for the corresponding generating function. In this way one generates a number of finite-dimensional solutions of the YBE including the trigonometric R-matrix (3.19) among them. More precisely, let us fix the spin parameters as $s_{1}=-\omega^{\prime \prime}-n_{1} \omega-m_{1} \omega^{\prime}, n_{1}, m_{1} \in$ $\mathbb{Z}_{\geq 0}$, and $s_{2}=-\omega^{\prime \prime}-n_{2} \omega-m_{2} \omega^{\prime}, n_{2}, m_{2} \in \mathbb{Z}_{\geq 0}$, in the first and second spaces, respectively. Then

$$
\begin{aligned}
& \mathbb{R}_{12}\left(u \mid s_{1}, s_{2}\right) \cdot D_{n_{1} \omega+m_{1} \omega^{\prime}}\left(x_{13}\right) D_{n_{2} \omega+m_{2} \omega^{\prime}}\left(x_{24}\right)=c \cdot D_{u-\frac{s_{1}}{2}-\frac{s_{2}}{2}}\left(x_{12}\right) D_{-u-\frac{s_{1}}{2}-\frac{s_{2}}{2}-\omega^{\prime \prime}}\left(x_{23}\right) \\
& \quad \times D_{n_{1} \omega+m_{1} \omega^{\prime}}\left(\hat{p}_{2}\right) \cdot D_{-u-\frac{s_{1}}{2}+\frac{s_{2}}{2}-\omega^{\prime \prime}}\left(x_{12}\right) D_{u-\frac{s_{1}}{2}+\frac{s_{2}}{2}}\left(x_{23}\right) D_{n_{2} \omega+m_{2} \omega^{\prime}}\left(x_{24}\right)
\end{aligned}
$$


is a concise expression for the finite-dimensional (in both spaces) R-matrix. After expansion with respect to auxiliary parameters $X_{3}\left(x_{3}\right), \widetilde{X}_{3}\left(x_{3}\right), X_{4}\left(x_{4}\right)$, and $\widetilde{X}_{4}\left(x_{4}\right)$ it can be rewritten explicitly is the form of an $\left(n_{1}+1\right)\left(m_{1}+1\right)\left(n_{2}+1\right)\left(m_{2}+1\right)$-dimensional matrix.

The integral R-operator exists as well for the $\mathcal{U}_{q}\left(\mathfrak{s l}_{2}\right)$-algebra [13], which is a "one-half" of the modular double. A reduction of this R-operator leads to the trigonometric L-operator as was shown in [9]. Derivation of the corresponding higher-spin finite-dimensional solutions of YBE using the described reduction procedure will be presented elsewhere.

\subsection{The fusion and symbols for $\mathcal{U}_{q}\left(\mathfrak{s l}_{2}\right)$ algebra}

Now we would like to show that the reduction result of the previous section can be derived with the help of the fusion procedure. In the present section we develop the fusion for the quantum algebra $\mathcal{U}_{q}\left(\mathfrak{s l}_{2}\right)$ and in the next one we consider the modular double. Our approach is not that well known since we extensively use the symbols of operators.

Similar to the discussion in Section 2.5 we construct the Lax operator with a finite-dimensional local quantum space out of the q-deformed Yang's R-matrix (remind that the deformation parameter and quasiperiods are related as $\left.q=e^{i \pi \omega / \omega^{\prime}}\right)$. The latter acts on the tensor product of two fundamental representations and is given by the matrix

$$
\begin{aligned}
\mathrm{R}(u) & =\frac{1}{2} \frac{q^{u+\frac{1}{2}}-q^{-u-\frac{1}{2}}}{q^{\frac{1}{2}}-q^{-\frac{1}{2}}} \mathbb{1}+\frac{1}{2} \sigma_{1} \otimes \sigma_{1}+\frac{1}{2} \sigma_{2} \otimes \sigma_{2}+\frac{1}{2} \frac{q^{u+\frac{1}{2}}+q^{-u-\frac{1}{2}}}{q^{\frac{1}{2}}+q^{-\frac{1}{2}}} \sigma_{3} \otimes \sigma_{3} \\
& =\left(\begin{array}{cc}
{\left[u+\frac{1}{2}+\frac{1}{2} \sigma_{3}\right]_{q}} & {\left[u+\frac{1}{2}-\frac{1}{2} \sigma_{3}\right]_{q}}
\end{array}\right)
\end{aligned}
$$

where $[x]_{q}$ is the $q$-number $[x]_{q}=\frac{q^{x}-q^{-x}}{q-q^{-1}}$. Here $\sigma_{ \pm}=\left(\sigma_{1} \pm i \sigma_{2}\right) / 2$ and the $q$-number of the matrix $\sigma_{3}$ is defined in an evident way, since it is diagonal.

In a full analogy with the non-deformed case, the recipe of $[28,29]$ suggests to form an inhomogeneous monodromy matrix out of the $q$-deformed Yang's R-matrices and to symmetrize it,

$$
\mathrm{R}_{\left(i_{1} \ldots i_{n}\right)}^{\left(j_{1} \ldots j_{n}\right)}(u):=\operatorname{Sym}_{i_{1}}^{j_{1}}(u) \mathrm{R}_{i_{1}}^{j_{1}}(u-1) \cdots \mathrm{R}_{i_{n}}^{j_{n}}(u-n+1)
$$

where Sym implies symmetrization with respect to indices $\left(i_{1} \ldots i_{n}\right)$ and $\left(j_{1} \ldots j_{n}\right)$ and all these indices refer to the first space of the R-matrix (3.38)

$$
\mathrm{R}_{i}^{j}(u)=\frac{1}{2} \frac{q^{u+\frac{1}{2}}-q^{-u-\frac{1}{2}}}{q^{\frac{1}{2}}-q^{-\frac{1}{2}}} \delta_{i}^{j}+\frac{1}{2}\left(\sigma_{1}\right)_{i}^{j} \otimes \sigma_{1}+\frac{1}{2}\left(\sigma_{2}\right)_{i}^{j} \otimes \sigma_{2}+\frac{1}{2} \frac{q^{u+\frac{1}{2}}+q^{-u-\frac{1}{2}}}{q^{\frac{1}{2}}+q^{-\frac{1}{2}}}\left(\sigma_{3}\right)_{i}^{j} \otimes \sigma_{3} .
$$

The standard way of treating (3.39) implies construction of the symmetrizer, i.e., a projector to the highest spin representation in the decomposition of the product of $n$ fundamental representations. We implement the projection by means of the auxiliary spinors $\lambda, \mu$ that is equivalent to dealing with the symbols of R-matrices. The symbol of (2.51) (with respect to the local quantum space, not the auxiliary one) factorizes (see (2.51))

$$
\begin{aligned}
\mathrm{R}(u \mid \lambda, \mu) & =\lambda_{i_{1}} \cdots \lambda_{i_{n}} \mathrm{R}_{i_{1} \ldots j_{n}}^{j_{1} \ldots j_{n}}(u) \mu_{j_{1}} \cdots \mu_{j_{n}} \\
& =\langle\lambda|\mathrm{R}(u)| \mu\rangle\langle\lambda|\mathrm{R}(u-1)| \mu\rangle \cdots\langle\lambda|\mathrm{R}(u-n+1)| \mu\rangle
\end{aligned}
$$

to a product of the symbols for $q$-Yang's R-matrices $\langle\lambda|\mathrm{R}(u)| \mu\rangle=\lambda_{i} \mathrm{R}_{i}^{j}(u) \mu_{j}$,

$$
\langle\lambda|\mathrm{R}(u)| \mu\rangle=\left(\begin{array}{cc}
{[u+1]_{q} \lambda_{1} \mu_{1}+[u]_{q} \lambda_{2} \mu_{2}} & \lambda_{2} \mu_{1} \\
\lambda_{1} \mu_{2} & {[u]_{q} \lambda_{1} \mu_{1}+[u+1]_{q} \lambda_{2} \mu_{2}}
\end{array}\right) .
$$


The product of $n$ such matrices is given by

$$
\mathrm{R}(u \mid \lambda, \mu)=[u]_{q}[u-1]_{q} \cdots[u-n+1]_{q}\left(\begin{array}{cc}
\mathrm{A}\left(u \mid \lambda_{1} \mu_{1}, \lambda_{2} \mu_{2}\right) & \mathrm{B}\left(\lambda_{1} \mu_{1}, \lambda_{2} \mu_{2}\right) \lambda_{2} \mu_{1} \\
\mathrm{~B}\left(\lambda_{2} \mu_{2}, \lambda_{1} \mu_{1}\right) \lambda_{1} \mu_{2} & \mathrm{~A}\left(u \mid \lambda_{2} \mu_{2}, \lambda_{1} \mu_{1}\right)
\end{array}\right),
$$

where the functions $A$ and $B$ have the following form

$$
\begin{aligned}
\mathrm{A}\left(u \mid \lambda_{1} \mu_{1}, \lambda_{2} \mu_{2}\right) & =\sum_{k=0}^{n} \frac{n !}{k !(n-k) !}[u+1+k-n]_{q}\left(\lambda_{1} \mu_{1}\right)^{n-k}\left(\lambda_{2} \mu_{2}\right)^{k} \\
& =\left[u+1-\frac{n}{2}+\frac{1}{2}\left(\lambda_{1} \partial_{\lambda_{1}}-\lambda_{2} \partial_{\lambda_{2}}\right)\right]_{q}\left(\lambda_{1} \mu_{1}+\lambda_{2} \mu_{2}\right)^{n}, \\
\mathrm{~B}\left(\lambda_{1} \mu_{1}, \lambda_{2} \mu_{2}\right)= & \sum_{k=0}^{n} \frac{n !}{k !(n-k) !}[k]_{q}\left(\lambda_{1} \mu_{1}\right)^{k-1}\left(\lambda_{2} \mu_{2}\right)^{n-k}= \\
& =\frac{1}{\lambda_{1} \mu_{1}}\left[\lambda_{1} \partial_{\lambda_{1}}\right]_{q}\left(\lambda_{1} \mu_{1}+\lambda_{2} \mu_{2}\right)^{n} .
\end{aligned}
$$

The summation formulae in (3.43), (3.44) facilitate reconstruction of operators from the symbolic entries of the matrix (3.42). In analogy with the non-deformed case we again remove the inessential normalization factor $r_{n}(u)=[u]_{q}[u-1]_{q} \cdots[u-n+1]_{q}$ and shift the spectral parameter $u \rightarrow u-1+\frac{n}{2}$ to obtain a symbol of the L-operator

$$
\begin{aligned}
\mathrm{L}(u \mid \lambda, \mu) & =r_{n}^{-1}(u) \mathrm{R}\left(u-1+\frac{n}{2} \mid \lambda, \mu\right) \\
& =\left(\begin{array}{cc}
\left.u+\frac{1}{2}\left(\lambda_{1} \partial_{\lambda_{1}}-\lambda_{2} \partial_{\lambda_{2}}\right)\right]_{q} & \frac{\lambda_{2}}{\lambda_{1}}\left[\lambda_{1} \partial_{\lambda_{1}}\right]_{q} \\
\frac{\lambda_{1}}{\lambda_{2}}\left[\lambda_{2} \partial_{\lambda_{2}}\right]_{q} & {\left[u+\frac{1}{2}\left(\lambda_{2} \partial_{\lambda_{2}}-\lambda_{1} \partial_{\lambda_{1}}\right)\right]_{q}}
\end{array}\right)\langle\lambda \mid \mu\rangle^{n} .
\end{aligned}
$$

Since $\langle\lambda \mid \mu\rangle^{n}$ is a symbol of the identity operator, applying (2.42), we immediately recover the familiar Lax operator,

$$
\mathrm{L}(u)=\left(\begin{array}{cc}
{\left[u+J_{3}\right]_{q}} & J_{-} \\
J_{+} & {\left[u-J_{3}\right]_{q}}
\end{array}\right)
$$

where the generators of $\mathcal{U}_{q}\left(\mathfrak{s l}_{2}\right)$ are realized by the finite-difference operators $J_{-}, J_{+}, q^{ \pm J_{3}}$ in two variables,

$$
J_{-}=\frac{\lambda_{2}}{\lambda_{1}}\left[\lambda_{1} \partial_{\lambda_{1}}\right]_{q}, \quad J_{+}=\frac{\lambda_{1}}{\lambda_{2}}\left[\lambda_{2} \partial_{\lambda_{2}}\right]_{q}, \quad J_{3}=\frac{1}{2}\left(\lambda_{1} \partial_{\lambda_{1}}-\lambda_{2} \partial_{\lambda_{2}}\right) .
$$

One can check that they do respect commutation relations of $\mathcal{U}_{q}\left(\mathfrak{s l}_{2}\right)$

$$
J_{+} J_{-}-J_{+} J_{-}=\left[2 J_{3}\right]_{q}, \quad J_{3} J_{ \pm}-J_{ \pm} J_{3}= \pm J_{ \pm} .
$$

Let us remind that the representation is defined on the space of homogeneous polynomials of two variables $\lambda_{1}, \lambda_{2}$ of degree $n$ (see (2.41)). In order to proceed to the space of polynomials of one variable we choose $\lambda_{1}=-z, \lambda_{2}=1$, so that the generators (3.46) take the conventional form

$$
J_{-}=-\frac{1}{z}[z \partial]_{q}, \quad J_{+}=z[z \partial-n]_{q}, \quad J_{3}=z \partial-\frac{n}{2} .
$$

We close this section by an alternative calculation of the symbol (3.40) which follows the pattern at the end of Section 2.5. The main merit of the following calculation is that it can be generalized to the elliptic case [10]. First of all we fix the auxiliary spinor $\lambda_{1}=-z, \lambda_{2}=1$ and use the realization of spin $\frac{1}{2}$ generators (cf. (3.47))

$$
J_{-}=-\frac{1}{z}[z \partial]_{q}, \quad J_{+}=z[z \partial-1]_{q}, \quad J_{3}=z \partial-\frac{1}{2},
$$


which act in the two-dimensional space of linear functions $\psi(z)=a_{1} z+a_{0}$. In the basis $\mathbf{e}_{1}=-z$, $\mathbf{e}_{2}=1$, the matrices of the generators coincide with the Pauli-matrices

$$
J_{ \pm}\left(\mathbf{e}_{1}, \mathbf{e}_{2}\right)=\left(\mathbf{e}_{1}, \mathbf{e}_{2}\right) \sigma_{ \pm}, \quad J_{3}\left(\mathbf{e}_{1}, \mathbf{e}_{2}\right)=\left(\mathbf{e}_{1}, \mathbf{e}_{2}\right) \frac{1}{2} \sigma_{3}
$$

The fusion procedure enables us to derive the L-operator (3.45) together with representation of the spin $\frac{n}{2}$ generators (3.47) acting in the $(n+1)$-dimensional space of polynomials $\psi(z)=$ $a_{n} z^{n}+\cdots+a_{0}$. Relations (3.48) are equivalent to

$$
(-z, 1) \sigma_{ \pm}=J_{ \pm}(-z, 1), \quad(-z, 1) \frac{1}{2} \sigma_{3}=J_{3}(-z, 1)
$$

that enables us to represent the symbol of $q$-Yang's R-matrix (3.38) as a matrix difference operator acting on the symbol of the identity operator (cf. (3.41)),

$$
\begin{aligned}
\langle\lambda|\mathrm{R}(u)| \mu\rangle & =\left(\begin{array}{cc}
(-z, 1)\left[u+\frac{1}{2}+\frac{1}{2} \sigma_{3}\right]_{q}|\mu\rangle & (-z, 1) \sigma_{-}|\mu\rangle \\
(-z, 1) \sigma_{+}|\mu\rangle & (-z, 1)\left[u+\frac{1}{2}-\frac{1}{2} \sigma_{3}\right]_{q}|\mu\rangle
\end{array}\right) \\
& =\left(\begin{array}{cc}
{[u+z \partial]_{q}} & -\frac{1}{z}[z \partial]_{q} \\
z[z \partial-1]_{q} & {[u+1-z \partial]_{q}}
\end{array}\right)\left(\mu_{2}-\mu_{1} z\right) .
\end{aligned}
$$

This operator is just the trigonometric L-operator (3.45) for the spin $\frac{1}{2}$ representation. The crucial observation is that it can be factorized respecting a special ordering of $z$ and $\partial$,

$$
\begin{aligned}
\langle\lambda|\operatorname{R}(u)| \mu\rangle= & \frac{1}{q-q^{-1}}\left(\begin{array}{cc}
1 & 1 \\
z q^{-u-1} & z q^{u+1}
\end{array}\right)\left(\begin{array}{cc}
q^{z_{1} \partial_{1}} & 0 \\
0 & q^{-z_{1} \partial_{1}}
\end{array}\right)\left(\begin{array}{cc}
q^{u} & -z^{-1} \\
-q^{-u} & z^{-1}
\end{array}\right) \\
& \times\left.\left(\mu_{2}-\mu_{1} z_{1}\right)\right|_{z_{1}=z} .
\end{aligned}
$$

Formula (3.49) represents a trigonometric deformation of the factorization formula (2.59).

The derived formula enables us to simplify the product of two consecutive symbols from (3.40) since a pair of adjacent matrix factors is cancelled

$$
\begin{aligned}
& \langle\lambda|\mathrm{R}(u)| \mu\rangle\langle\lambda|\mathrm{R}(u-1)| \mu\rangle=\frac{1}{\left(q-q^{-1}\right)^{2}}\left(\begin{array}{cc}
1 & 1 \\
z q^{-u-1} & z q^{u+1}
\end{array}\right)\left(\begin{array}{cc}
q^{z_{1} \partial_{1}} & 0 \\
0 & q^{-z_{1} \partial_{1}}
\end{array}\right) \\
& \quad \times\left(\begin{array}{cc}
q^{u} & -z^{-1} \\
-q^{-u} & z^{-1}
\end{array}\right)\left(\begin{array}{cc}
1 & 1 \\
z q^{-u} & z q^{u}
\end{array}\right)\left(\begin{array}{cc}
q^{z_{2} \partial_{2}} & 0 \\
0 & q^{-z_{2} \partial_{2}}
\end{array}\right)\left(\begin{array}{cc}
q^{u-1} & -z^{-1} \\
-q^{-u+1} & z^{-1}
\end{array}\right) \\
& \quad \times\left.\left(\mu_{2}-\mu_{1} z_{1}\right)\left(\mu_{2}-\mu_{1} z_{2}\right)\right|_{z_{1}=z_{2}=z}=\frac{[u]_{q}}{q-q^{-1}}\left(\begin{array}{cc}
1 & 1 \\
z q^{-u-1} & z q^{u+1}
\end{array}\right) \\
& \quad \times\left.\left(\begin{array}{cc}
q^{z_{1} \partial_{1}+z_{2} \partial_{2}} & 0 \\
0 & q^{-z_{1} \partial_{1}-z_{2} \partial_{2}}
\end{array}\right)\left(\begin{array}{cc}
q^{u-1} & -z^{-1} \\
-q^{-u+1} & z^{-1}
\end{array}\right)\left(\mu_{2}-\mu_{1} z_{1}\right)\left(\mu_{2}-\mu_{1} z_{2}\right)\right|_{z_{1}=z_{2}=z} .
\end{aligned}
$$

The generalization of this formula is obvious. Thus the product of symbols (3.40) is equal to

$$
\begin{aligned}
\mathrm{R}(u \mid \lambda, \mu)= & r_{n}(u) \frac{1}{q-q^{-1}}\left(\begin{array}{cc}
1 & 1 \\
z q^{-u-1} & z q^{u+1}
\end{array}\right)\left(\begin{array}{cc}
q^{z_{1} \partial_{1}+\cdots+z_{n} \partial_{n}} & 0 \\
0 & q^{-z_{1} \partial_{1}-\cdots-z_{n} \partial_{n}}
\end{array}\right) \\
& \times\left.\left(\begin{array}{cc}
q^{u-n+1} & -z^{-1} \\
-q^{-u+n-1} & z^{-1}
\end{array}\right)\left(\mu_{2}-\mu_{1} z_{1}\right) \cdots\left(\mu_{2}-\mu_{1} z_{n}\right)\right|_{z_{1}=\cdots=z_{n}=z} .
\end{aligned}
$$

Then we need to get rid off the taken special ordering in (3.50). To that end we apply an evident formula

$$
\left.q^{ \pm\left(z_{1} \partial_{1}+\cdots+z_{n} \partial_{n}\right)}\left(\mu_{2}-\mu_{1} z_{1}\right) \cdots\left(\mu_{2}-\mu_{1} z_{n}\right)\right|_{z_{1}=\cdots=z_{n}=z}=q^{ \pm z \partial}\left(\mu_{2}-\mu_{1} z\right)^{n}
$$


which enables us to cast the matrix product on the right-hand side of $(3.50)$ in the form

$$
\begin{aligned}
r_{n}(u) & \left(\begin{array}{cc}
\left.u-n+1+z_{1} \partial_{1}+\cdots+z_{n} \partial_{n}\right]_{q} & -\frac{1}{z}\left[z_{1} \partial_{1}+\cdots+z_{n} \partial_{n}\right]_{q} \\
z\left[z_{1} \partial_{1}+\cdots+z_{n} \partial_{n}-n\right]_{q} & {\left[u+1-z_{1} \partial_{1}-\cdots-z_{n} \partial_{n}\right]_{q}}
\end{array}\right) \\
& \times\left.\left(\mu_{2}-\mu_{1} z_{1}\right) \cdots\left(\mu_{2}-\mu_{1} z_{n}\right)\right|_{z_{1}=\cdots=z_{n}=z} \\
= & r_{n}(u)\left(\begin{array}{cc}
{[u-n+1+z \partial]_{q}} & -\frac{1}{z}[z \partial]_{q} \\
z[z \partial-n]_{q} & {[u+1-z \partial]_{q}}
\end{array}\right)\left(\mu_{2}-\mu_{1} z\right)^{n} .
\end{aligned}
$$

Thus the final result for the symbol of the "fused" $q$-Yang R-matrices (3.39) is

$$
\mathrm{R}(u \mid \lambda, \mu)=r_{n}(u)\left(\begin{array}{cc}
\left.u+1-\frac{n}{2}+J_{3}\right]_{q} & J_{-} \\
J_{+} & {\left[u+1-\frac{n}{2}-J_{3}\right]_{q}}
\end{array}\right)\left(\mu_{2}-\mu_{1} z\right)^{n},
$$

where the generators $J_{ \pm}, J_{3}$ in spin $\frac{n}{2}$ representation are given by the expression (3.47).

\subsection{Fusion construction for the modular double}

The fusion procedure for the modular double closely follows the construction from Section 2.6. One forms inhomogeneous monodromy matrix out of the L-operators and then symmetrizes it over the spinor indices resulting in a finite-dimensional (in one of the spaces) higher-spin L-operator.

Again, instead of working with the higher-rank tensors we introduce auxiliary spinors $\lambda_{i}$, $\widetilde{\lambda}_{i}, \mu_{j}$, and $\widetilde{\mu}_{j}$ and contract them with the monodromy matrix according to (2.40). The homogeneity (2.41) implies that there are redundant variables. We get rid off them by choosing the gauge $\lambda_{1} \lambda_{2}=-1, \mu_{1} \mu_{2}=-1$ that is equivalent to the parametrization of the spinors by means of the independent variables $a$ and $b$ as follows

$$
\begin{array}{ll}
\lambda_{1}=\lambda_{1}(a)=e^{\frac{i \pi}{2 \omega} a}, & \lambda_{2}=\lambda_{2}(a)=-e^{-\frac{i \pi}{2 \omega} a}, \\
\mu_{1}=\mu_{1}(b)=e^{\frac{i \pi}{2 \omega} b}, & \mu_{2}=\mu_{2}(b)=-e^{-\frac{i \pi}{2 \omega} b} .
\end{array}
$$

Analogous relations hold for the spinors $\widetilde{\lambda}$ and $\widetilde{\mu}$ obtained from (3.51) after the interchange $\omega \rightleftarrows \omega^{\prime}$ with the same $a$ and $b$. Since we assume that the ratio of quasiperiods $\tau$ is not rational, $\lambda$ and $\widetilde{\lambda}$ are multiplicatively incommensurate for generic $a$ and the same is true for $\mu$ and $\widetilde{\mu}$ for generic $b$.

Further, we form symbols of the L-operators (3.18) (i.e., some scalar operators) contracting them in the matrix space with the auxiliary spinors ${ }^{2}$

$$
\lambda_{i} \mathrm{~L}_{i}^{j}(u) \mu_{j}=\Lambda(u, \lambda, \mu), \quad \widetilde{\lambda}_{i} \widetilde{\mathrm{L}}_{i}^{j}(u) \widetilde{\mu}_{j}=\widetilde{\Lambda}(u, \widetilde{\lambda}, \widetilde{\mu}) .
$$

Taking into account that $D_{\omega^{\prime}}(\hat{p})=e^{-\frac{i \pi}{2 \omega} \hat{p}}+e^{\frac{i \pi}{2 \omega} \hat{p}}$ (see (3.5), (3.6)), one can easily check the equality

$$
D_{u_{2}+\omega^{\prime}}(x-a) D_{-u_{1}}(x+b) D_{\omega^{\prime}}(\hat{p}) D_{-u_{2}}(x-a) D_{u_{1}+\omega^{\prime}}(x+b)=i \Lambda(u) .
$$

It will be helpful to rewrite this formula in a slightly different form by means of the operator star-triangle relation (3.31)

$$
D_{u_{2}+\omega^{\prime}}(x-a) D_{u_{1}+\omega^{\prime}}(\hat{p}) D_{\omega^{\prime}}(x+b) D_{-u_{1}}(\hat{p}) D_{-u_{2}}(x-a)=i \Lambda(u) .
$$

\footnotetext{
${ }^{2}$ We are grateful to D. Karakhanyan and R. Kirschner for a discussion on this point.
} 
The analogous relation is valid for $\widetilde{\Lambda}(u)$, which is obtained after the permutation $\omega \rightleftarrows \omega^{\prime}$. The derived formula is reminiscent to the L-operator factorization (3.20). Now we form a string out of the symbols $\Lambda$ and $\widetilde{\Lambda}$ (3.53) with the shifted spectral parameters,

$$
\begin{aligned}
\mathrm{R}_{\text {fus }}(u \mid \lambda, \widetilde{\lambda}, \mu, \widetilde{\mu})= & \Lambda(u) \Lambda\left(u-\omega^{\prime}\right) \cdots \Lambda\left(u-(m-1) \omega^{\prime}\right) \\
& \times \widetilde{\Lambda}\left(u-(m-1) \omega^{\prime}-\omega\right) \cdots \widetilde{\Lambda}\left(u-(m-1) \omega^{\prime}-n \omega\right) .
\end{aligned}
$$

In view of the reflection formula (3.7) and relation (3.53) this product can be recast to the form

$$
\begin{aligned}
\mathrm{R}_{\text {fus }}(u \mid \lambda, \widetilde{\lambda}, \mu, \widetilde{\mu})= & D_{u_{2}+\omega^{\prime}}(x-a) D_{u_{1}+\omega^{\prime}}(\hat{p})\left[i D_{\omega^{\prime}}(x+b)\right]^{m}\left[i D_{\omega}(x+b)\right]^{n} \\
& \times D_{-u_{1}+(m-1) \omega^{\prime}+n \omega}(\hat{p}) D_{-u_{2}+(m-1) \omega^{\prime}+n \omega}(x-a) .
\end{aligned}
$$

Finally, we reconstruct the operator of interest from its symbol using formula (2.42), which results in the representation

$$
\left[\mathrm{R}_{\text {fus }}(u) \Phi\right](\lambda, \widetilde{\lambda} \mid x)=\left.\mathrm{R}_{\text {fus }}\left(u \mid \lambda, \widetilde{\lambda}, \partial_{\mu}, \partial_{\widetilde{\mu}}\right) \Phi(\mu, \widetilde{\mu} \mid x)\right|_{\mu=\widetilde{\mu}=0},
$$

where the symbol $R_{\text {fus }}$ is fixed in (3.54). Let us stress once more that the fusion formula (3.55) is completely analogous to the $\mathrm{SL}(2, \mathbb{C})$ group case $(2.63)$. The higher-spin R-operator acts on a function $\Phi(\lambda, \widetilde{\lambda} \mid x)$ having the homogeneity degrees $m$ in $\lambda$ and $n$ in $\widetilde{\lambda}$, respectively. In (3.55) one has differentiations over spinors $\mu, \widetilde{\mu}$, but the operator $\mathrm{R}_{\text {fus }}(3.54)$ formally depends on $b$ and not on the exponential of $b$. In order to see that there is no contradiction, we note that according to the definitions (3.52) $\Lambda$ and $\widetilde{\Lambda}$ are linear in spinors. Consequently $R_{\text {fus }}(3.54)$, being a product of them, has to be polynomial in spinors. This can be checked directly as well. Recalling the definition of $\mu, \widetilde{\mu}(3.51)$ and

$$
D_{\omega^{\prime}}(x+b)=\mu_{1} e^{\frac{i \pi}{2 \omega} x}-\mu_{2} e^{-\frac{i \pi}{2 \omega} x}, \quad D_{\omega}(x+b)=\widetilde{\mu}_{1} e^{\frac{i \pi}{2 \omega^{\prime}} x}-\widetilde{\mu}_{2} e^{-\frac{i \pi}{2 \omega^{\prime}} x},
$$

we conclude that $\mathrm{R}_{\text {fus }}$ in (3.54) depends polynomially on $\mu$ and $\widetilde{\mu}$. Thus the fusion formulae (3.54) and (3.55) match to each other.

The right-hand side of (3.54) explicitly depends on $a$, so its polynomiality in spinors $\lambda, \widetilde{\lambda}$ is not obvious at all. It is necessary to demonstrate it explicitly. Furthermore, we need to compare (3.55) with the reduction formula (3.36), since both give rise to a higher-spin R-operator. We will accomplish both tasks if we show that the R-operators do coincide. Thus we take the generating function $D_{n \omega+m \omega^{\prime}}(a-y)$ of a finite-dimensional representation and act upon it by the "fused" R-operator in the first space according to the prescription (3.55). The generating function with the auxiliary parameter $y$ explicitly depends on $\lambda$ and $\widetilde{\lambda}$ (see (3.15)),

$$
\begin{aligned}
D_{n \omega+m \omega^{\prime}}(a-y)= & \prod_{k=0}^{n-1}\left(\widetilde{\lambda}_{1} e^{-\frac{i \pi}{2 \omega^{\prime}} y} \tilde{q}^{\frac{n-1}{2}-k}-(-1)^{m} \widetilde{\lambda}_{2} e^{\frac{i \pi}{2 \omega^{\prime}} y} \tilde{q}^{-\frac{n-1}{2}+k}\right) . \\
& \times \prod_{p=0}^{m-1}\left(\lambda_{1} e^{-\frac{i \pi}{2 \omega} y} q^{\frac{m-1}{2}-p}-(-1)^{n} \lambda_{2} e^{\frac{i \pi}{2 \omega} y} q^{-\frac{m-1}{2}+p}\right),
\end{aligned}
$$

and has the homogeneity degrees $m$ in $\lambda$ and $n$ in $\tilde{\lambda}$, respectively. Now, using the relations (see $(3.56),(3.57))$

$$
\left.\left[D_{\omega^{\prime}}(x+b)\right]^{m}\left[D_{\omega}(x+b)\right]^{n}\right|_{\mu \rightarrow \partial_{\mu}, \widetilde{\mu} \rightarrow \partial_{\widetilde{\mu}}} D_{n \omega+m \omega^{\prime}}(b-y)=n ! m ! D_{n \omega+m \omega^{\prime}}(x-y),
$$

we can perform differentiations over spinors in (3.55) that immediately yield the desired result

$$
\begin{aligned}
& \mathrm{R}_{\text {fus }}\left(u+\frac{n \omega}{2}+\frac{m \omega^{\prime}}{2}\right) D_{n \omega+m \omega^{\prime}}(a-y)=i^{n+m} n ! m ! D_{u-\frac{s_{0}}{2}-\frac{s}{2}}(x-a) D_{u-\frac{s_{0}}{2}+\frac{s}{2}}(\hat{p}) \\
& \quad \times D_{n \omega+m \omega^{\prime}}(x-y) D_{-u-\frac{s_{0}}{2}-\frac{s}{2}-\omega^{\prime \prime}}(\hat{p}) D_{-u-\frac{s_{0}}{2}+\frac{s}{2}-\omega^{\prime \prime}}(x-a)=i^{n+m} n ! m ! D_{u-\frac{s_{0}}{2}-\frac{s}{2}}(x-a) \\
& \quad \times D_{-u-\frac{s_{0}}{2}-\frac{s}{2}-\omega^{\prime \prime}}(x-y) D_{n \omega+m \omega^{\prime}}(\hat{p}) D_{u-\frac{s_{0}}{2}+\frac{s}{2}}(x-y) D_{-u-\frac{s_{0}}{2}+\frac{s}{2}-\omega^{\prime \prime}}(x-a),
\end{aligned}
$$


where at the last step we profited from the operator star-triangle relation (3.31). Identifying the variables $a=x_{1}, x=x_{2}, y=x_{3}$, we find a nice agreement of the fusion formula (3.59) with the reduction formula (3.36). Thus both approaches are equivalent and yield identical results.

\section{Acknowledgement}

We thank the referees for useful remarks to the paper. This work is supported by the Russian Science Foundation (project no. 14-11-00598).

\section{References}

[1] Baxter R.J., Exactly solved models in statistical mechanics, Academic Press, Inc., London, 1982.

[2] Bazhanov V.V., Mangazeev V.V., Sergeev S.M., Faddeev-Volkov solution of the Yang-Baxter equation and discrete conformal symmetry, Nuclear Phys. B $\mathbf{7 8 4}$ (2007), 234-258, hep-th/0703041.

[3] Bazhanov V.V., Stroganov Yu.G., Chiral Potts model as a descendant of the six-vertex model, J. Statist. Phys. 59 (1990), 799-817.

[4] Bytsko A.G., Teschner J., R-operator, co-product and Haar-measure for the modular double of $U_{q}(\mathfrak{s l}(2, \mathbb{R}))$, Comm. Math. Phys. 240 (2003), 171-196, math.QA/0208191.

[5] Bytsko A.G., Teschner J., Quantization of models with non-compact quantum group symmetry: modular $X X Z$ magnet and lattice sinh-Gordon model, J. Phys. A: Math. Gen. 39 (2006), 12927-12981, hep-th/0602093.

[6] Chicherin D., Derkachov S., The R-operator for a modular double, J. Phys. A: Math. Theor. 47 (2014), 115203, 14 pages, arXiv:1309.0803.

[7] Chicherin D., Derkachov S., Matrix factorization for solutions of the Yang-Baxter equation, Zap. Nauchn. Semin. POMI 433 (2015), 156-185, arXiv:1502.07923.

[8] Chicherin D., Derkachov S., Karakhanyan D., Kirschner R., Baxter operators for arbitrary spin, Nuclear Phys. B 854 (2012), 393-432, arXiv:1106.4991.

[9] Chicherin D., Derkachov S., Karakhanyan D., Kirschner R., Baxter operators with deformed symmetry, Nuclear Phys. B $\mathbf{8 6 8}$ (2013), 652-683, arXiv:1211.2965.

[10] Chicherin D., Derkachov S.E., Spiridonov V.P., New elliptic solutions of the Yang-Baxter equation, Comm. Math. Phys., to appear, arXiv:1412.3383.

[11] Chicherin D., Spiridonov V.P., The hyperbolic modular double and Yang-Baxter equation, Adv. Stud. Pure Math., to appear, arXiv:1511.00131.

[12] De Vega H.J., Lipatov L.N., Interaction of Reggeized gluons in the Baxter-Sklyanin representation, Phys. Rev. D 64 (2001), 114019, 27 pages, hep-ph/0107225.

[13] Derkachov S., Karakhanyan D., Kirschner R., Yang-Baxter $\mathcal{R}$-operators and parameter permutations, Nuclear Phys. B $\mathbf{7 8 5}$ (2007), 263-285, hep-th/0703076.

[14] Derkachov S.E., Korchemsky G.P., Manashov A.N., Noncompact Heisenberg spin magnets from highenergy QCD. I. Baxter Q-operator and separation of variables, Nuclear Phys. B 617 (2001), 375-440, hep-th/0107193.

[15] Derkachov S.E., Manashov A.N., A general solution of the Yang-Baxter equation with the symmetry group $\mathrm{SL}(n, \mathbb{C})$, St. Petersburg Math. J. 21 (2010), 513-577.

[16] Derkachov S.E., Spiridonov V.P., The Yang-Baxter equation, parameter permutations, and the elliptic beta integral, Russ. Math. Surv. 68 (2013), 1027-1072, arXiv:1205.3520.

[17] Derkachov S.E., Spiridonov V.P., Finite-dimensional representations of the elliptic modular double, Theoret. and Math. Phys. 183 (2015), 597-618, arXiv:1310.7570.

[18] Faddeev L., Modular double of a quantum group, in Conférence Moshé Flato 1999, Vol. I (Dijon), Math. Phys. Stud., Vol. 21, Kluwer Acad. Publ., Dordrecht, 2000, 149-156, math.QA/9912078.

[19] Faddeev L.D., Discrete Heisenberg-Weyl group and modular group, Lett. Math. Phys. 34 (1995), 249-254, hep-th/9504111.

[20] Faddeev L.D., Kashaev R.M., Volkov A.Yu., Strongly coupled quantum discrete Liouville theory. I. Algebraic approach and duality, Comm. Math. Phys. 219 (2001), 199-219, hep-th/0006156. 
[21] Faddeev L.D., Korchemsky G.P., High-energy QCD as a completely integrable model, Phys. Lett. B 342 (1995), 311-322, hep-th/9404173.

[22] Furlan P., Ganchev A.Ch., Petkova V.B., Fusion matrices and $c<1$ (quasi) local conformal theories, Internat. J. Modern Phys. A 5 (1990), 2721-2735.

[23] Gel'fand I.M., Graev M.I., Vilenkin N.Ya., Generalized functions, Vol. 5. Integral geometry and representation theory, Academic Press, New York - London, 1966.

[24] Gel'fand I.M., Nămark M.A., Unitary representations of the Lorentz group, Trudy Mat. Inst. Steklov., Vol. 36, Izdat. Nauk SSSR, Moscow - Leningrad, 1950.

[25] Isaev A.P., Multi-loop Feynman integrals and conformal quantum mechanics, Nuclear Phys. B 662 (2003), 461-475, hep-th/0303056.

[26] Jimbo M. (Editor), Yang-Baxter equation in integrable systems, Advanced Series in Mathematical Physics, Vol. 10, World Sci. Publ. Co., Inc., Teaneck, NJ, 1989.

[27] Khoroshkin S., Tsuboi Z., The universal $R$-matrix and factorization of the $L$-operators related to the Baxter Q-operators, J. Phys. A: Math. Theor. 47 (2014), 192003, 11 pages, arXiv:1401.0474.

[28] Kulish P.P., Reshetikhin N.Yu., Sklyanin E.K., Yang-Baxter equations and representation theory. I, Lett. Math. Phys. 5 (1981), 393-403.

[29] Kulish P.P., Sklyanin E.K., Quantum spectral transform method. Recent developments, Springer, Berlin New York, 1982, 61-119.

[30] Lipatov L.N., High-energy asymptotics of multicolor QCD and two-dimensional conformal field theories, Phys. Lett. B 309 (1993), 394-396.

[31] Lipatov L.N., High-energy asymptotics of multicolor QCD and exactly solvable lattice models, JETP Lett. 59 (1994), 596-599, hep-th/9311037.

[32] Mangazeev V.V., On the Yang-Baxter equation for the six-vertex model, Nuclear Phys. B 882 (2014), 70-96, arXiv:1401.6494.

[33] Mangazeev V.V., Q-operators in the six-vertex model, Nuclear Phys. B 886 (2014), 166-184, arXiv:1406.0662.

[34] Pawelkiewicz M., Schomerus V., Suchanek P., The universal Racah-Wigner symbol for $U_{q}(\operatorname{osp}(1 \mid 2))$, J. High Energy Phys. 2014 (2014), no. 4, 079, 26 pages, arXiv:1307.6866.

[35] Ponsot B., Teschner J., Clebsch-Gordan and Racah-Wigner coefficients for a continuous series of representations of $\mathcal{U}_{q}(\mathfrak{s l}(2, \mathbb{R}))$, Comm. Math. Phys. 224 (2001), 613-655, math.QA/0007097.

[36] Spiridonov V.P., Essays on the theory of elliptic hypergeometric functions, Russ. Math. Surv. 63 (2008), 405-472, arXiv:0805.3135.

[37] Spiridonov V.P., The continuous biorthogonality of an elliptic hypergeometric function, St. Petersburg Math. J. 20 (2009), 791-812, arXiv:0801.4137.

[38] Spiridonov V.P., Elliptic beta integrals and solvable models of statistical mechanics, in Algebraic Aspects of Darboux Transformations, Quantum Integrable Systems and Supersymmetric Quantum Mechanics, Contemp. Math., Vol. 563, Amer. Math. Soc., Providence, RI, 2012, 181-211, arXiv:1011.3798.

[39] Tarasov V.O., Takhtadzhyan L.A., Faddeev L.D., Local Hamiltonians for integrable quantum models on a lattice, Theoret. and Math. Phys. 57 (1983), 1059-1073.

[40] van de Bult F.J., Hyperbolic hypergeometric functions, Ph.D. Thesis, University of Amsterdam, 2007.

[41] Vasil'ev A.N., The field theoretic renormalization group in critical behavior theory and stochastic dynamics, Chapman \& Hall/CRC, Boca Raton, FL, 2004.

[42] Volkov A.Yu., Noncommutative hypergeometry, Comm. Math. Phys. 258 (2005), 257-273, math.QA/0312084.

[43] Volkov A.Yu., Faddeev L.D., Yang-baxterization of a quantum dilogarithm, J. Math. Sci. 88 (1995), 202207. 